\title{
Evaluation of shear and membrane locking in refined hierarchical shell finite elements for laminated structures
}

Guohong Li ${ }^{1 *} \mathbb{D}$, Erasmo Carrera ${ }^{1,2}$, Maria Cinefra ${ }^{1}$, Alberto G. de Miguel ${ }^{1}$, Gennady M. Kulikov², Alfonso Pagani ${ }^{1}$ and Enrico Zappino ${ }^{1}$

${ }^{*}$ Correspondence: guohong.li@polito.it ${ }^{1}$ MUL2 Group, Department of Mechanical and Aerospace Engineering, Politecnico di Torino, Corso Duca degli Abruzzi 24, 10129 Turin, Italy

Full list of author information is available at the end of the article

\begin{abstract}
Shear and membrane locking phenomena are fundamental issues of shell finite element models. A family of refined shell elements for laminated structures has been developed in the framework of Carrera Unified Formulation, including hierarchical elements based on higher-order Legendre polynomial expansions. These hierarchical elements were reported to be relatively less prone to locking phenomena, yet an exhaustive evaluation of them regarding the mitigation of shear and membrane locking on laminated shells is still essential. In the present article, numerically efficient integration schemes for hierarchical elements, including also reduced and selective integration procedures, are discussed and evaluated through single-element $p$-version finite element models. Both shear and membrane locking are assessed quantitatively through the estimation of strain energy components. The numerical results show that the fully integrated hierarchical shell elements can overcome the shear and membrane locking effectively when a sufficiently high polynomial degree is reached. Reduced and selective integration schemes can help with the mitigation of locking on lower-order hierarchical shell elements.
\end{abstract}

Keywords: Shell models, Hierarchical elements, Carrera Unified Formulation, Shear locking, Membrane locking

\section{Introduction}

Shell structures, especially composite laminated shells, have been widely used in modern engineering due to their high efficiency in holding loads. A variety of shell theories have been proposed, including the Classical Lamination Theory (CLT) based on Kirchhoff-Love hypothesis [1-3], the First-Order Deformation Theory (FSDT) based on the Mindlin-Reissner assumption [4,5], a series of Higher-Order Theories (HOT) [6-8], and a variety of refined shell theories generated in the framework of Carrera Unified Formulation (CUF) [9]. Refined shell theories have been implemented in the Finite Element (FE) method and can provide solutions with great accuracy $[10,11]$. Towards implementing numerically efficient simulation methods for multi-layered structures, hierarchical functions have been adopted in the construction of refined shell elements in the framework of CUF [12]. Nevertheless, the effectiveness of hierarchical functions concerning the

(c) The Author(s) 2019. This article is distributed under the terms of the Creative Commons Attribution 4.0 International License (http://creativecommons.org/licenses/by/4.0/), which permits unrestricted use, distribution, and reproduction in any medium, provided you give appropriate credit to the original author(s) and the source, provide a link to the Creative Commons license, and indicate if changes were made. 
mitigation of shear and membrane locking on refined multi-layered shell finite elements remains to be quantitatively assessed.

The locking phenomena are caused by the greatly overestimated stiffness of thin structures and will lead to a loss of convergence rate of the numerical solution. If no treatment is introduced, the meshes of the shell FE models have to be immensely refined, which makes the analysis numerically prohibitive. Shear locking, caused by the so-called "parasitic shear" in the bending of a thin shell, is a typical locking phenomenon [13]. Due to the incompetence of the shell elements in capturing the bending deformation appropriately, the strain energy is absorbed by the shear mode erroneously. As the shell structures become thinner, the transverse shear energy approaches zero, physically. On the other hand, membrane locking can be observed on shell elements when bending deformation is incorrectly accompanied by the stretching of the mid-surface, and the membrane energy overshadows the bending part $[14,15]$.

Pioneering simple remedies to the locking phenomena are the reduced integration and selectively reduced integration techniques $[13,16]$. Zienkiewicz et al. [13] pointed out that by reducing the order of numerical integration, the stiffness of displacement-based finite elements can be decreased. The main idea of selective integration is to reduce the shear stiffness, and the reduced quadrature is selectively used on the stiffness component related to transverse shear. This method is reported to be useful in bending problems yet was found less effective compared with uniformly reduced integration on all the stiffness components for general shell problems [13]. This reduced integration approach brings significant improvement to the convergence rate. The equivalence of the reduced integration procedure with mixed formulation was demonstrated by Malkus and Hughes [16] and Zienkiewicz and Nakazawa [17].

A drawback of the reduced integration technique is the introduction of "spurious modes" due to the erroneously evaluated stiffness matrix. A typical example is the "hour-glass" mode of four-node bi-linear shell element with reduced integration. Zienkiewicz and Taylor [18] commented that for general applications mixed elements are preferred than reduced integration procedures. This numerical singularity problem can also be avoided by using alternative techniques such as the Mixed Interpolation of Tensorial Components (MITC) proposed by Bathe et al. [19-22]. In the MITC formulation, the shear locking can be overcome by the additional independent interpolation functions for the transverse shear strains. This approach is also referred to as the "assumed shear strain field" method [23]. The link between MITC formulation and the Hellinger-Reissner mixed variation principle was demonstrated by Bathe et al. [22]. The mathematical justification of MITC formulation was established through the Babuska-Brezzi conditions [24]. In the framework of CUF, MITC has been successfully applied to build locking-free refined elements with variable kinematics for multi-layered plates by Carrera et al. [25-27] and for shell structures by Cinefra and Valvano [11] and Carrera et al. [26]. An extension of MITC technique to beam elements was also addressed by Carrera et al. [28]. Very recent developments of four-node MITC elements were presented by Ko et al. [29,30].

Indeed, shear locking effects are more pronounced on low-order elements [31]. The loss of convergence can be alleviated by adopting higher order elements [32,33], such as higherorder hierarchical elements $[34,35]$. A combination of hierarchical elements and mixed interpolation method was proposed and applied to isotropic plates based on ReissnerMindlin assumption by Scapolla and Della Croce [36,37]. An application of hierarchi- 


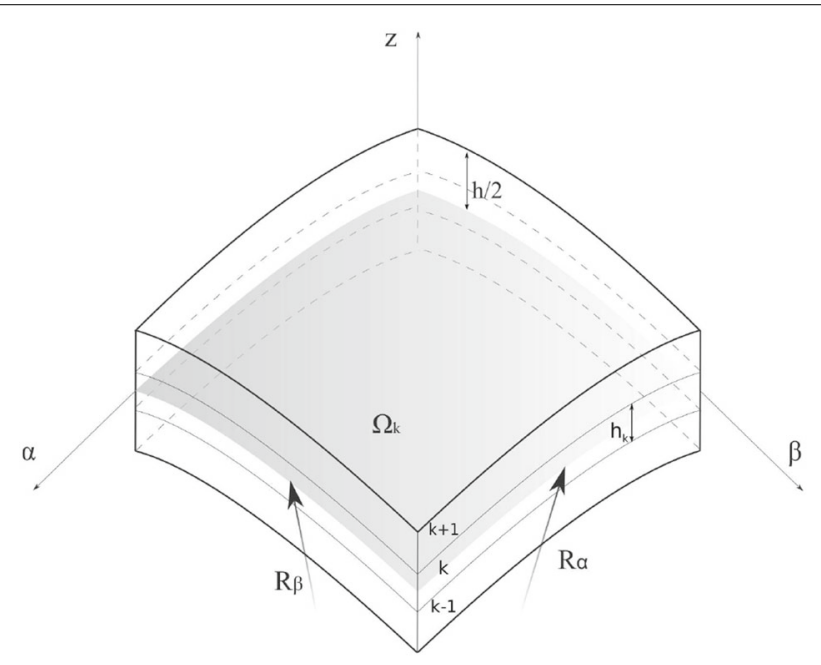

Fig. 1 Notation of a shell model for laminated structures

cal elements using Naghdi shell model on isotropic structures was reported by Chinosi et al. [38]. The selective and reduced integration schemes on hierarchical elements were primarily discussed by Della Croce and Scapolla [39]. Carrera et al. [12] reported a comparison between shell elements with MITC and plain hierarchical shape functions in the analysis of laminated structures.

The numerical efficiency of the hierarchical elements has been reported by many researchers $[34,35,40,41]$. In the framework of CUF, this type of hierarchical functions has been used on refined beam [42], plate [43], and shell [12] finite element models for multi-layered structures. Via refined hierarchical 2D elements, the FE models can be mathematically enriched on both the kinematic and shape function levels, leading to an adaptable refinement FE approach with optimal numerical efficiency. This article aims to present the evaluation of hierarchical elements concerning the mitigation of shear and membrane locking phenomena in the analysis of multi-layered shells. In the following sections, we first introduce the multi-layered shell models and CUF briefly. Then, an energy decomposition method is presented for refined shell models based on CUF. Thirdly, numerically efficient full, reduced, and selective integration schemes are discussed. Finally, the mitigation of shear locking and membrane locking is demonstrate through two numerical examples, respectively. The numerical evaluations are compared against available analytical solutions in the literature.

\section{Refined shell finite element formulation}

Preliminaries of multi-layered shell model

Figure 1 shows a typical laminated shell structure with curvatures. This geometry can be described in the orthogonal curvilinear reference system $(\alpha, \beta, z)$, in which $\alpha$ and $\beta$ indicate the two in-plane directions and $z$ the through-thickness direction which is usually measured with reference to the middle surface. The infinitesimal in-plane area $d S$ and volume $d V$ can be written as:

$$
\begin{aligned}
d S & =H_{\alpha} H_{\beta} d \alpha d \beta=H_{\alpha} H_{\beta} d \Omega, \\
d V & =H_{\alpha} H_{\beta} H_{z} d \alpha d \beta d z .
\end{aligned}
$$


where $d \Omega$ is the infinitesimal in-plane area on the middle surface, and $H_{\alpha}, H_{\beta}$ and $H_{z}$ are:

$$
H_{\alpha}=A\left(1+z / R_{\alpha}\right), \quad H_{\beta}=B\left(1+z / R_{\beta}\right), \quad H_{z}=1 .
$$

in which $R_{\alpha}$ and $R_{\beta}$ are the principal radii of the middle surface, $A$ and $B$ the coefficients of the first fundamental form of $\Omega$. The present work considers only shells with constant curvatures, for which $A=B=1$. For more details about shell formulations, the reader is referred to $[44,45]$.

The strains and stresses defined in the curvilinear reference system are:

$$
\begin{aligned}
\boldsymbol{\epsilon} & =\left\{\epsilon_{\alpha \alpha}, \epsilon_{\beta \beta}, \epsilon_{z z}, \epsilon_{\alpha z}, \epsilon_{\beta z}, \epsilon_{\alpha \beta}\right\}^{T} \\
\sigma & =\left\{\sigma_{\alpha \alpha}, \sigma_{\beta \beta}, \sigma_{z z}, \sigma_{\alpha z}, \sigma_{\beta z}, \sigma_{\alpha \beta}\right\}^{T}
\end{aligned}
$$

The strains $\epsilon$ can be obtained through the geometrical relations:

$$
\epsilon=b u
$$

wherein $\boldsymbol{u}=\{u, v, w\}^{T}$ is the displacement vector, and $\boldsymbol{b}$ the differential operators matrix, whose explicit expression reads:

$$
\boldsymbol{b}=\left[\begin{array}{ccc}
\frac{\partial_{\alpha}}{H_{\alpha}} & 0 & \frac{1}{H_{\alpha} R_{\alpha}} \\
0 & \frac{\partial_{\beta}}{H_{\beta}} & \frac{1}{H_{\beta} R_{\beta}} \\
0 & 0 & \partial_{z} \\
\partial_{z}-\frac{1}{H_{\alpha} R_{\alpha}} & 0 & \frac{\partial_{\alpha}}{H_{\alpha}} \\
0 & \partial_{z}-\frac{1}{H_{\beta} R_{\beta}} & \frac{\partial_{\beta}}{H_{\beta}} \\
\frac{\partial_{\beta}}{H_{\beta}} & \frac{\partial_{\alpha}}{H_{\alpha}} & 0
\end{array}\right]
$$

The stresses can be attained from the constitutive equations as follows:

$$
\sigma=C \epsilon
$$

in which $C$ is the material coefficients matrix which is obtained by transforming the original form $\boldsymbol{C}_{0}$ from the material coordinate $\operatorname{system}(1,2,3)$ to the global system $(\alpha, \beta, z)$. The orthotropic material coefficients are characterized by nine independent coefficients, namely Young's moduli, shear moduli, and Poisson ratios [7].

\section{Carrera Unified Formulation (CUF) for refined 2D models}

Through Carrera Unified Formulation (CUF), the displacement field of a shell structure can be assumed as:

$$
\boldsymbol{u}(\alpha, \beta, z)=F_{\tau}(z) \boldsymbol{u}_{\tau}(\alpha, \beta)
$$

where $\boldsymbol{u}_{\tau}(\alpha, \beta)$ are the in-plane displacement vectors, and functions $F_{\tau}(z)$ are related to the theories of structures (TOS). Since $F_{\tau}(z)$ depends only on the thickness coordinates, they are also referred to as thickness functions. By increasing the polynomial order of these thickness functions, the shell kinematic models can be refined. Both EquivalentSingle-Layer (ESL) and Layer-Wise (LW) models can be accounted for in this framework, as elaborated by Carrera et al. [46]. The FSDT [47] can be treated as a particular case of the 
HOT models adopting Taylor expansions (TE). In the LW model framework, Lagrangian polynomial expansions (LE) can be used to formulate kinematics with only translational degrees of freedom. More discussions about these two types of refined kinematic models can be found in the work of Carrera et al. [46]. By using LE, the interfacial continuity of transverse stresses can be approximately achieved when the thickness functions are adequately refined, as demonstrated by Carrera et al. [26].

When the FE discretization is introduced, the in-plane displacements of a shell structure are approximated through the shape functions $N_{i}(\alpha, \beta)$ through:

$$
\boldsymbol{u}_{\tau}(\alpha, \beta)=N_{i}(\alpha, \beta) \boldsymbol{u}_{i \tau}
$$

in which $\boldsymbol{u}_{i \tau}$ are nodal unknown variables. The above expression leads to FE formulation in the framework of CUF:

$$
\begin{aligned}
\boldsymbol{u}(\alpha, \beta, z) & =F_{\tau}(z) N_{i}(\alpha, \beta) \boldsymbol{u}_{i \tau} \\
\delta \boldsymbol{u}(\alpha, \beta, z) & =F_{s}(z) N_{j}(\alpha, \beta) \delta \boldsymbol{u}_{j s}
\end{aligned}
$$

where $\delta$ indicates the virtual variation. The above expression is compact through the use of repeated indexes. By applying the Principle of Virtual Displacements (PVD), the general expressions of the stiffness matrix and load vector of the FE model, namely the Fundamental Neuclei (FNs), can be obtained. The explicit expressions of the FNs are given in [12]. As expounded by Carrera et al. [46], the CUF-type FE formulation is independent of the kinematic assumptions adopted and is a general framework for the development of refined FE models. For more details about the derivation of shell FE formulations in the framework of CUF, the reader is referred to the work of Carrera et al. [46].

\section{Decomposition of strain energy in refined shell models}

For a general laminated shell structure, the strain energy can be decomposed into four parts as follows:

$$
\begin{aligned}
E_{p n} & =\frac{1}{2} \int_{V}\left(\varepsilon_{\alpha \alpha} \sigma_{\alpha \alpha}+\varepsilon_{\beta \beta} \sigma_{\beta \beta}\right) d V \\
E_{p s} & =\frac{1}{2} \int_{V} \varepsilon_{\alpha \beta} \sigma_{\alpha \beta} d V \\
E_{z s} & =\frac{1}{2} \int_{V}\left(\varepsilon_{\alpha z} \sigma_{\alpha z}+\varepsilon_{\beta z} \sigma_{\beta z}\right) d V \\
E_{z z} & =\frac{1}{2} \int_{V} \varepsilon_{z z} \sigma_{z z} d V
\end{aligned}
$$

where $E_{p n}$ represents the in-plane normal energy, $E_{p s}$ the in-plane shear energy, $E_{z s}$ the transverse shear energy, and $E_{z z}$ the thickness stretch energy. The transverse shear energy allows us to evaluate the shear locking effects in shell elements. The introduction of the thickness stretch energy makes it convenient to assess the performance of the adopted structural theory. Note that the above decomposition applies to arbitrarily laminated shell structures.

To calculate the strain energy components, their corresponding stiffness matrices are necessary. These matrices can be obtained through standard FE procedure in the framework of CUF. Taking the transverse shear energy $E_{z s}$ as an example, by recalling the PVD, one has:

$$
\delta E_{z s}=\int_{V}\left(\delta \varepsilon_{\alpha z} \sigma_{\alpha z}+\delta \varepsilon_{\beta z} \sigma_{\beta z}\right) d V=\delta \boldsymbol{u}_{j s} \cdot \boldsymbol{k}_{i j \tau s}^{z s} \cdot \boldsymbol{u}_{i \tau}
$$


wherein $\boldsymbol{k}_{i j \tau s}^{z s}$ is the FNs for the transverse shear stiffness matrix of the element. By substituting the displacement approximations (Eq. 11) into the geometrical relations (Eq. 6), and considering the constitutive equations (Eq. 8), one obtains:

$$
\begin{aligned}
& \left\{\begin{array}{l}
\delta \varepsilon_{\alpha z} \\
\delta \varepsilon_{\beta z}
\end{array}\right\}=\boldsymbol{b}_{z s} \cdot \delta \boldsymbol{u}=\left[\begin{array}{cccc}
\partial_{z}-\frac{1}{H_{\alpha} R_{\alpha}} & 0 & \frac{\partial_{\alpha}}{H_{\alpha}} \\
0 & \partial_{z}-\frac{1}{H_{\beta} R_{\beta}} & \frac{\partial_{\beta}}{H_{\beta}}
\end{array}\right] \cdot N_{j} F_{s} \delta \boldsymbol{u}_{j s} \\
& \left\{\begin{array}{c}
\sigma_{\alpha z} \\
\sigma_{\beta z}
\end{array}\right\}=\boldsymbol{C}_{z s} \cdot \boldsymbol{\varepsilon}=\left[\begin{array}{llllll}
C_{41} & C_{42} & C_{43} & C_{44} & C_{45} & C_{46} \\
C_{51} & C_{52} & C_{53} & C_{54} & C_{55} & C_{56}
\end{array}\right] \cdot\left(\boldsymbol{b} N_{i} F_{\tau} \boldsymbol{u}_{i \tau}\right)
\end{aligned}
$$

thus the FNs for the transverse shear stiffness matrix $\boldsymbol{k}_{i t \tau s}^{z s}$ can be obtained as:

$$
\boldsymbol{k}_{i j \tau s}^{z s}=\int_{V}\left(\boldsymbol{b}_{z s}^{T} N_{j} F_{s}\right) \boldsymbol{C}_{z s}\left(\boldsymbol{b} N_{i} F_{\tau}\right) d V
$$

Through the assembly of $\boldsymbol{k}_{i j \tau s}^{z s}$ according to the standard procedure of FE formulation in CUF framework, the transverse shear stiffness matrix $\boldsymbol{K}_{z s}$ can be obtained. When the displacement solutions are obtained, the transverse shear strain energy can be calculated through:

$$
E_{z s}=\frac{1}{2} \int_{V}\left(\varepsilon_{\alpha z} \sigma_{\alpha z}+\varepsilon_{\beta z} \sigma_{\beta z}\right) d V=\frac{1}{2} \boldsymbol{u}^{T} \cdot \boldsymbol{K}_{z s} \cdot \boldsymbol{u}
$$

The in-plane normal stiffness matrix $\boldsymbol{K}_{p n}$, in-plane shear stiffness matrix $\boldsymbol{K}_{p s}$, and outof-plane normal stiffness matrix $\boldsymbol{K}_{z z}$ can be achieved accordingly by means of the following FNs:

$$
\begin{aligned}
\boldsymbol{k}_{i j \tau s}^{p n} & =\int_{V}\left(\boldsymbol{b}_{p n}^{T} N_{j} F_{s}\right) \boldsymbol{C}_{p n}\left(\boldsymbol{b} N_{i} F_{\tau}\right) d V \\
\boldsymbol{k}_{i j \tau s}^{p s} & =\int_{V}\left(\boldsymbol{b}_{p s}^{T} N_{j} F_{s}\right) \boldsymbol{C}_{p s}\left(\boldsymbol{b} N_{i} F_{\tau}\right) d V \\
\boldsymbol{k}_{i j \tau s}^{z z} & =\int_{V}\left(\boldsymbol{b}_{z z}^{T} N_{j} F_{s}\right) \boldsymbol{C}_{z z}\left(\boldsymbol{b} N_{i} F_{\tau}\right) d V
\end{aligned}
$$

wherein $\boldsymbol{b}_{p n}, \boldsymbol{b}_{p s}$, and $\boldsymbol{b}_{z z}$ are the sub-matrices of the differential operators matrix $\boldsymbol{b}$ as in Eq. 7, and their explicit expressions are:

$$
\begin{aligned}
\boldsymbol{b}_{p n} & =\left[\begin{array}{ccc}
\frac{\partial_{\alpha}}{H_{\alpha}} & 0 & \frac{1}{H_{\alpha} R_{\alpha}} \\
0 & \frac{\partial_{\beta}}{H_{\beta}} & \frac{1}{H_{\beta} R_{\beta}}
\end{array}\right] \\
\boldsymbol{b}_{p s} & =\left[\begin{array}{lll}
\frac{\partial_{\beta}}{H_{\beta}} & \frac{\partial_{\alpha}}{H_{\alpha}} & 0
\end{array}\right] \\
\boldsymbol{b}_{z z} & =\left[\begin{array}{lll}
0 & 0 & \partial_{z}
\end{array}\right]
\end{aligned}
$$

and their corresponding material coefficients matrices (sub-matrices of the material coefficients matrix $\boldsymbol{C}$ ) are as follows:

$$
\begin{aligned}
C_{p n} & =\left[\begin{array}{llllll}
C_{11} & C_{12} & C_{13} & C_{14} & C_{15} & C_{16} \\
C_{21} & C_{22} & C_{23} & C_{24} & C_{25} & C_{26}
\end{array}\right] \\
C_{p s} & =\left[\begin{array}{llllll}
C_{61} & C_{62} & C_{63} & C_{64} & C_{65} & C_{66}
\end{array}\right] \\
C_{z z} & =\left[\begin{array}{llllll}
C_{31} & C_{32} & C_{33} & C_{34} & C_{35} & C_{36}
\end{array}\right]
\end{aligned}
$$

In the end, the complete stiffness FNs can be obtained as the summation of these terms as:

$$
\boldsymbol{k}_{i j \tau s}=\boldsymbol{k}_{i j \tau s}^{p n}+\boldsymbol{k}_{i j \tau s}^{p s}+\boldsymbol{k}_{i j \tau s}^{z s}+\boldsymbol{k}_{i j \tau s}^{z z}
$$


If the multi-layered shell has symmetric lamination properties, the neutral surface of bending will coincide with the geometrical middle surface, and the in-plane normal strain energy, as in Eq. 12, can be further decomposed into membrane energy $E_{m e m b}$ and bending energy $E_{\text {bend }}$ conveniently through:

$$
\begin{aligned}
E_{\text {memb }} & =\frac{1}{2} \int_{V}\left(\varepsilon_{\alpha \alpha}^{0} \sigma_{\alpha \alpha}+\varepsilon_{\beta \beta}^{0} \sigma_{\beta \beta}\right) d V \\
E_{\text {bend }} & =\frac{1}{2} \int_{V}\left[\left(\varepsilon_{\alpha \alpha}-\varepsilon_{\alpha \alpha}^{0}\right) \sigma_{\alpha \alpha}+\left(\varepsilon_{\beta \beta}-\varepsilon_{\beta \beta}^{0}\right) \sigma_{\beta \beta}\right] d V
\end{aligned}
$$

wherein $\varepsilon_{\alpha \alpha}^{0}$ and $\varepsilon_{\beta \beta}^{0}$ are the normal strains due to the mid-surface straining, and they can be attained by means of:

$$
\left\{\begin{array}{c}
\varepsilon_{\alpha \alpha}^{0} \\
\varepsilon_{\beta \beta}^{0}
\end{array}\right\}=\boldsymbol{b}_{p n} \cdot \boldsymbol{u}=\left[\begin{array}{ccc}
\frac{\partial_{\alpha}}{H_{\alpha}} & 0 & \frac{1}{H_{\alpha} R_{\alpha}} \\
0 & \frac{\partial_{\beta}}{H_{\beta}} & \frac{1}{H_{\beta} R_{\beta}}
\end{array}\right] \cdot N_{j} F_{s}(0) \boldsymbol{u}_{j s}
$$

By following the procedure described before, $\boldsymbol{k}_{i j \tau s}^{m e m b}$, the FNs for the membrane stiffness matrix $\boldsymbol{K}_{m e m b}$, can be derived. The bending energy can be then obtained through:

$$
E_{\text {bend }}=E_{p n}-E_{\text {memb }}
$$

This separation of membrane and bending energy components provides the convenience to evaluate the existence of membrane locking and better understand the structural responses. It should be noted that $E_{p n}$ and $E_{p s}$ are both in-plane strain energy components, however since in laminated plates and shells the calculation $E_{p s}$ does not dependent on a specific neutral surface as the membrane and bending energy components do, it is considered apart in the present article.

\section{Integration schemes for hierarchical elements}

This section addresses the efficient integration schemes of 2D hierarchical elements with full, reduced, and selectively reduced integration. According to Szabó et al. [35,41], the hierarchical shape functions for $2 \mathrm{D}$ elements can be classified into nodal modes, edge modes, and surface modes, which can be expressed in a unified manner as:

$$
N_{i}(\xi, \eta)=\phi_{m}(\xi) \phi_{n}(\eta) \quad(\xi, \eta) \in[-1,1]
$$

in which the basis polynomials $\phi_{m}(\xi)$ and $\phi_{n}(\eta)$ are decided by their corresponding mode and the polynomial degree, as it is illustrated in Fig. 2.

In the FNs of stiffness matrix as given by Li et al. [12], the contribution of the shape functions to the stiffness matrix accounts for the following integrals:

$$
\begin{array}{lrl}
\triangleleft N_{i} N_{j} \triangleright \Omega, & \triangleleft N_{i} N_{j, \alpha} \triangleright \Omega, & \triangleleft N_{i} N_{j, \beta} \triangleright_{\Omega}, \\
\triangleleft N_{i, \alpha} N_{j} \triangleright \Omega, & \triangleleft N_{i, \beta} N_{j} \triangleright \Omega, & \triangleleft N_{i, \alpha} N_{j, \alpha} \triangleright_{\Omega}, \\
\triangleleft N_{i, \alpha} N_{j, \beta} \triangleright_{\Omega}, & \triangleleft N_{i, \beta} N_{j, \alpha} \triangleright \Omega, & \triangleleft N_{i, \beta} N_{j, \beta} \triangleright_{\Omega} .
\end{array}
$$

where $\triangleleft \cdots \triangleright_{\Omega}$ represents $\int_{\Omega} \cdots d \xi d \eta$. Among these terms, for given $i$ and $j$ combination, the polynomials with the highest order is $N_{i} \cdot N_{j}$. Consider the product of $N_{i}$ and $N_{j}$ :

$$
N_{i}(\xi, \eta) \cdot N_{j}(\xi, \eta)=\phi_{m}(\xi) \phi_{n}(\eta) \cdot \phi_{r}(\xi) \phi_{s}(\eta)=\phi_{m}(\xi) \phi_{r}(\xi) \cdot \phi_{n}(\eta) \phi_{s}(\eta)
$$

Thus in the $\xi$ and $\eta$ directions, the highest polynomial orders are $m+r$ and $n+s$, respectively. For simplicity, in the present work, the same set of Gauss points are used to calculate the above integrals of given $N_{i}$ and $N_{j}$. 


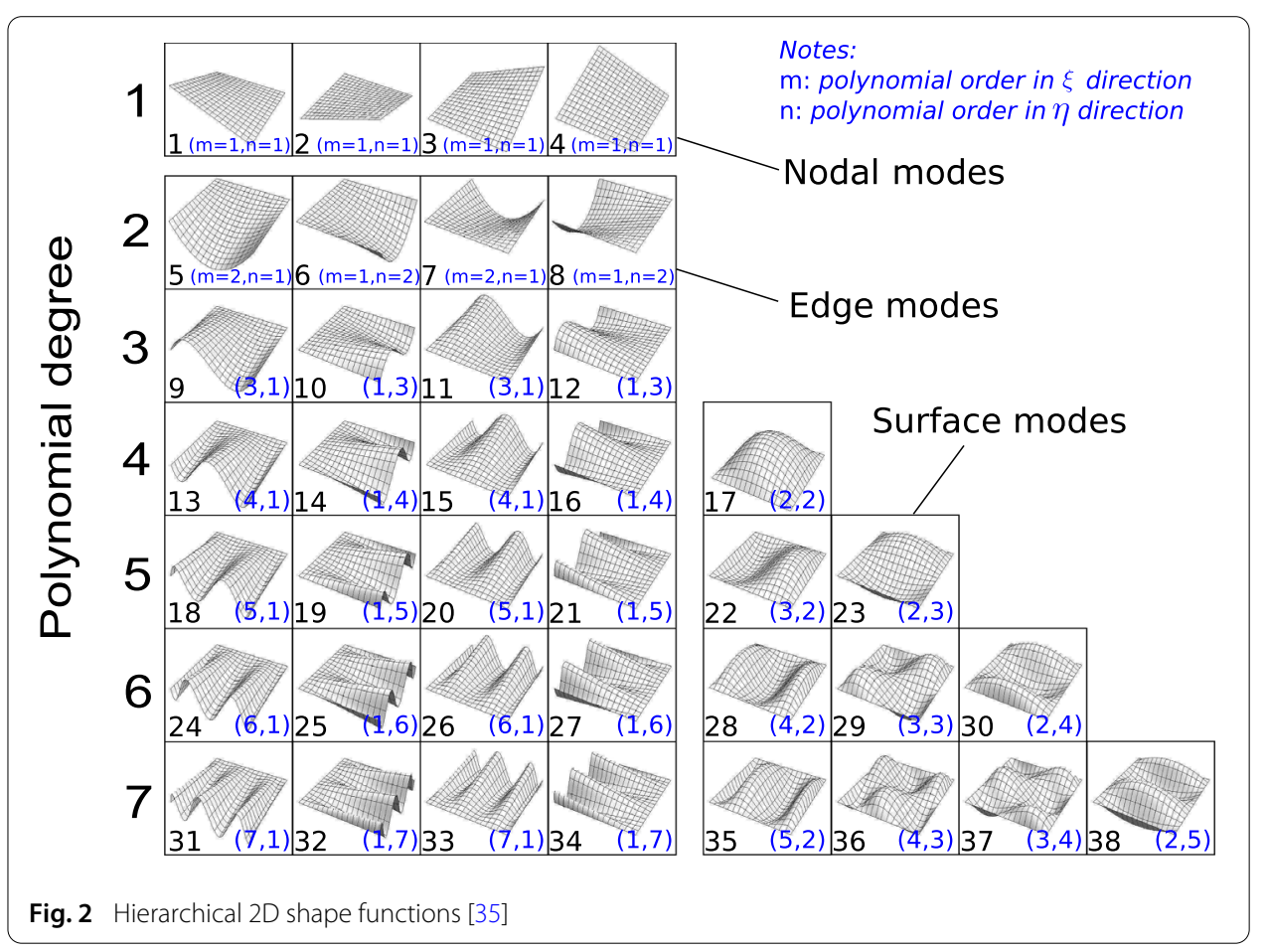

\section{Full integration scheme}

In Gauss-Legendre quadrature, $n$ Gauss points guarantee the exact integration of a polynomial of order $2 n-1$. For the exact integration of $\triangleleft N_{i} N_{j} \triangleright \Omega$, the least number of Gauss points used in the $\xi$ direction, $N_{G X}$, should be:

$$
N_{G X}= \begin{cases}(m+r) / 2+1, & \text { if } m+r=2 N \\ (m+r+1) / 2, & \text { if } m+r=2 N+1\end{cases}
$$

wherein $N$ is an arbitrary positive integer. The above expression also applies to the calculation of number of Gauss points in the $\eta$ direction, $N_{G Y}$, for an exact integration.

For classical Lagrangian elements, since all the shape functions have the same polynomial order in both $\xi$ and $\eta$ directions, the scheme of Gauss points can be uniformly decided. Differently, for hierarchical elements, the required number of Gauss points varies according to different combinations of shape functions. Also, in practice, for given $N_{i}$ and $N_{j}$, the same set of Gauss integration points can be used for the nine integrals in Eq. 36, which is determined by the highest polynomial order given by $N_{i} \cdot N_{j}$. Figure 3 presents two examples of the Gauss points distribution when full integration is used on hierarchical elements. Figure 3a shows the numerical calculation of $\triangleleft N_{13} \cdot N_{14} \triangleright \Omega$ needs $3 \times 3$ Gauss points. $\triangleleft N_{13} \cdot N_{15} \triangleright \Omega$ requires $5 \times 2$ Gauss points for its full integration, as illustrated in Fig. 3b.

\section{Reduced integration scheme}

The reduced integration technique on the hierarchical elements can be used in the following manners: applying the reduced integration to polynomials with the highest order among all $N_{i} \cdot N_{j}$ combinations, and using full integration for all the lower-order polyno- 

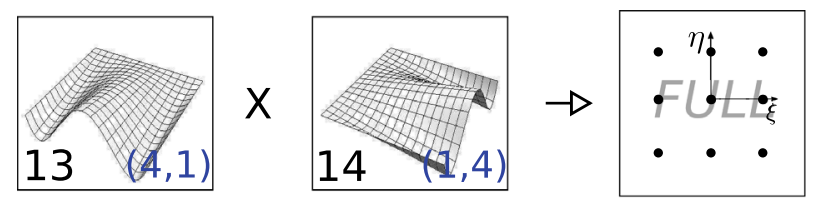

(a) $\triangleleft N_{13} \cdot N_{14} \triangleright \Omega$
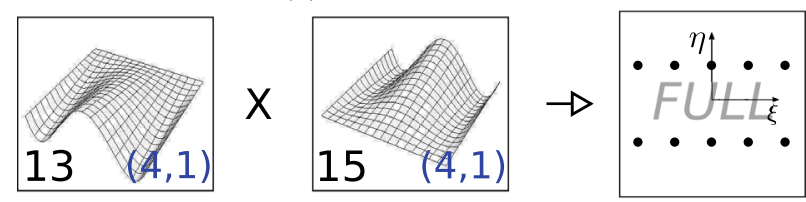

(b) $\triangleleft N_{13} \cdot N_{15} \triangleright \Omega$

Fig. 3 Gauss points used for the full integration of hierarchical shell elements. "FULL" represents the adoption of the full integration approach

mials. In the hierarchical element with polynomial degree $p$, as shown in Fig. $2, N_{i} \cdot N_{j}$ with the highest orders is a combination of the edge modes:

$$
N_{i} \cdot N_{j}= \begin{cases}\phi_{p}(\xi) \phi_{1}(\eta) \cdot \phi_{p}(\xi) \phi_{1}(\eta), & \text { in } \xi \text { direction; } \\ \phi_{1}(\xi) \phi_{p}(\eta) \cdot \phi_{1}(\xi) \phi_{p}(\eta), & \text { in } \eta \text { direction. }\end{cases}
$$

where the highest order of the polynomials to be integrated is $2 p$, and $p$ Gauss points are needed for the reduced integration. The product polynomials to be integrated in the other direction are of the second order and are fully integrated by using two Gauss points. Meanwhile, all the lower-order terms should be exactly integrated.

The hierarchical element with $p=4$ can be taken as an example. The polynomials to be integrated with the highest order in the $\xi$ direction are $N_{13} \cdot N_{13}, N_{13} \cdot N_{15}, N_{15} \cdot N_{13}$, and $N_{15} \cdot N_{15}$. Those with the highest order in the $\eta$ direction are $N_{14} \cdot N_{14}, N_{14} \cdot N_{16}$, $N_{16} \cdot N_{14}$, and $N_{16} \cdot N_{16}$. When the reduced integration scheme is adopted, $4 \times 2$ Gauss points should be used for the first group of polynomials (see Fig. 4a), and a $2 \times 4$ mesh of Gauss points for the second set (see Fig. 4b). For this fourth-order hierarchical element, the integration schemes that should be used for different blocks have been indicated in Fig. 5. Note that each block represented by a square is a sub-matrix $\boldsymbol{K}_{i j}$.

\section{Selectively reduced integration scheme}

In the selectively reduced integration method, the low-order integration is only applied to those terms related to the transverse shear stiffness. This technique is aimed to reduce the transverse shear stiffness to alleviate the shear locking phenomenon. These components can be determined by considering the FNs of the transverse shear stiffness matrix in Eq. 19. Also, the sub-matrices of the stiffness matrix $\boldsymbol{K}_{i j}$ that should be selectively integrated follow the same rule as the reduced integration technique as discussed in the "Reduced integration scheme" section. 

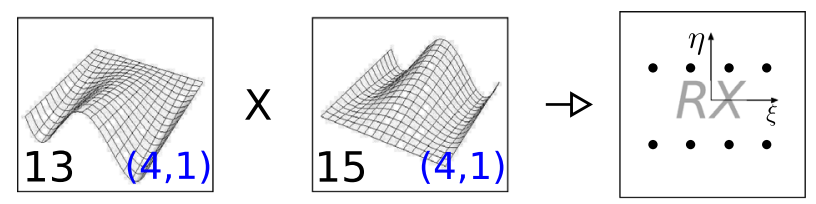

(a) $\triangleleft N_{13} \cdot N_{15} \triangleright_{\Omega}$
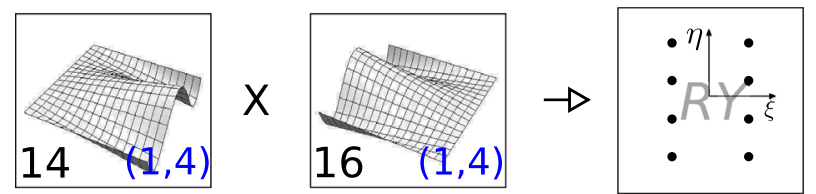

(b) $\triangleleft N_{14} \cdot N_{16} \triangleright_{\Omega}$

Fig. 4 Gauss points used for the reduced integration of hierarchical shell elements with $p=4$. "RX" and "RY" indicate reduced integration in the $\xi$ and $\eta$ directions, respectively

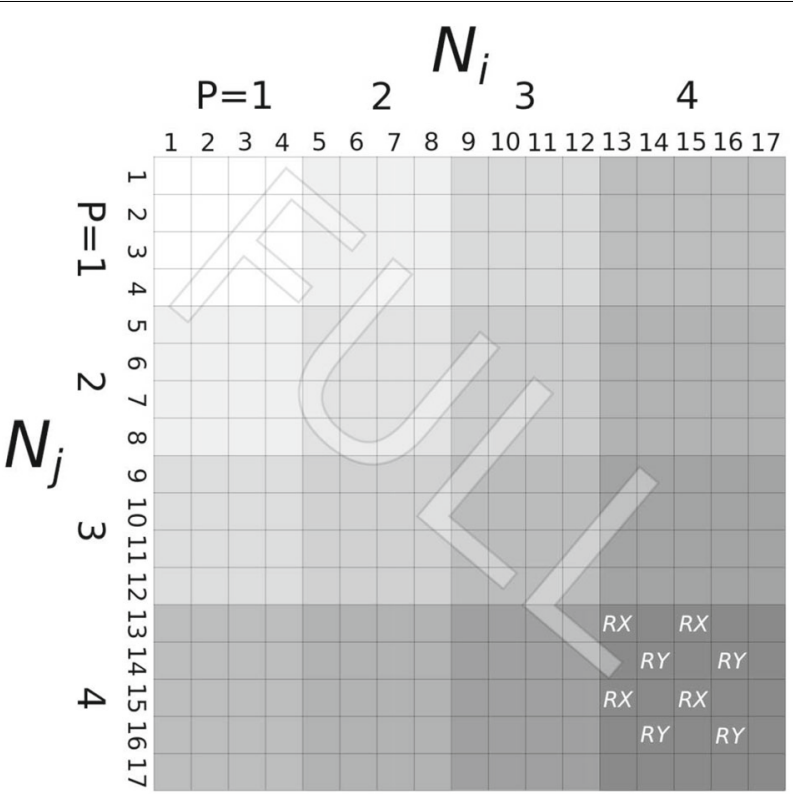

Fig. 5 Reduced integration scheme for an hierarchical element with $p=4$. "RX": reduced integration in the $\xi$ direction; "RY": reduced integration in the $\eta$ direction; "FULL": full integration in both directions

\section{Properties of the stiffness matrix of hierarchical elements with reduced and selective} integration

When reduced and selective integration techniques are used on low-order Lagrangian elements, spurious modes may occur. In this section, the eigenvalues of hierarchical elements with reduced and selective integration are calculated respectively to examine the properties of their stiffness matrices.

The adopted FE models consist of only one element. This square element has the inplane geometry of $1 \times 1$ and contains only one layer with thickness $h=1$. The used isotropic material has $E=10^{9}$ and $v=0.3$. The kinematic model (TOS) chosen is LE1 (LW model with Lagrangian first-order polynomials). A plate model without curvatures is used which is adequate for the examination of the shape function properties. 


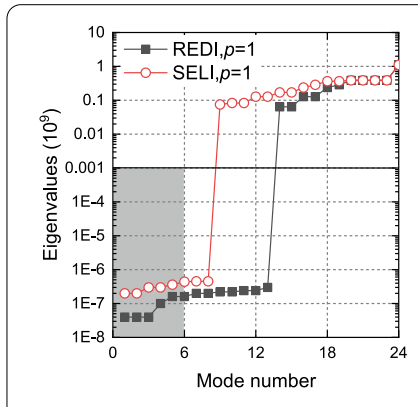

(a) $p=1$

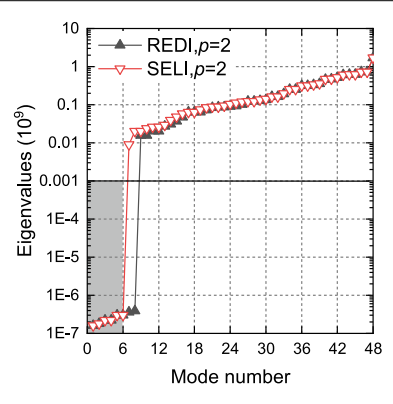

(b) $p=2$

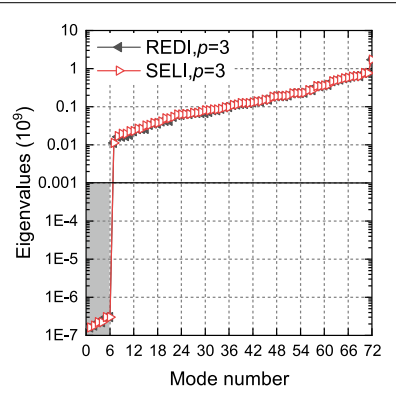

(c) $p=3$

Fig. 6 Eigenvalues of hierarchical elements with reduced integration (REDI) and selective integration (SELI)

Figure 6 reports the eigenvalues of the stiffness matrices of hierarchical elements $(p=$ $1,2,3)$ with reduced and selective integration. Note that hierarchical elements with $p=1$ are equivalent to standard Q4 (four-node quadrilateral Lagrangian) element. Figure 6a shows that when $p=1$, both reduced and selective integration schemes lead to more than six zero eigenvalues numerically, which means the elements are not robust. From Fig. 6b, it can be observed that for polynomial degree $p=2$, the reduced integration leads to two spurious modes (which is equal to the number of thickness functions used), and the element with selective integration has exactly six rigid-body modes. When the polynomial degree is further increased to $p=3$, the spurious modes are eliminated on the elements with reduced integration, see Fig. $6 \mathrm{c}$. The results demonstrate that, it can be guaranteed that there is zero spurious mode for the reduced integrated hierarchical elements when $p \geq 3$, and no spurious mode exists for selective integration when $p \geq 2$.

\section{Results and discussion}

This section presents two numerical examples on laminated shells considering a wide range of aspect ratio. Single-element FE models are used with $p$-version refinements. It is obvious that an element with only linear shape functions is not adequate for the modeling, thus the refinement of the shape functions starts from $p=2$. The polynomial degree is increased till the chosen convergence threshold is reached. Two kinds of TOS are used in the numerical modeling, namely the FSDT and LE4 (LW model with fourth-order Lagrange polynomials in each layer, see Carrera et al. [46]). These two theories are compared through elements with full integration. Then, with LE4 theory, different integration techniques on the hierarchical elements are compared, including full integration (FULL), reduced integration (REDI), and selective integration (SELI). Besides the displacement and stress evaluations, the strain energy components are also reported. The numerical results are compared against available analytical reference solutions.

\section{Three-layered cylindrical shells under distributed pressure}

Three-layered cylindrical shells with symmetric lamination $\left(0^{\circ} / 90^{\circ} / 0^{\circ}\right)$ are studied. The analytical solutions were presented by Varadan and Bhaskar [48]. The three layers have equal thickness $h / 3$. Radius-to-thickness ratios $R_{\beta} / h$ ranging from 2 to 500 are investigated. The cylindrical shells are simply supported on the two ends and subjected to transverse distributed pressure on the inner surface. The load distribution follows: 


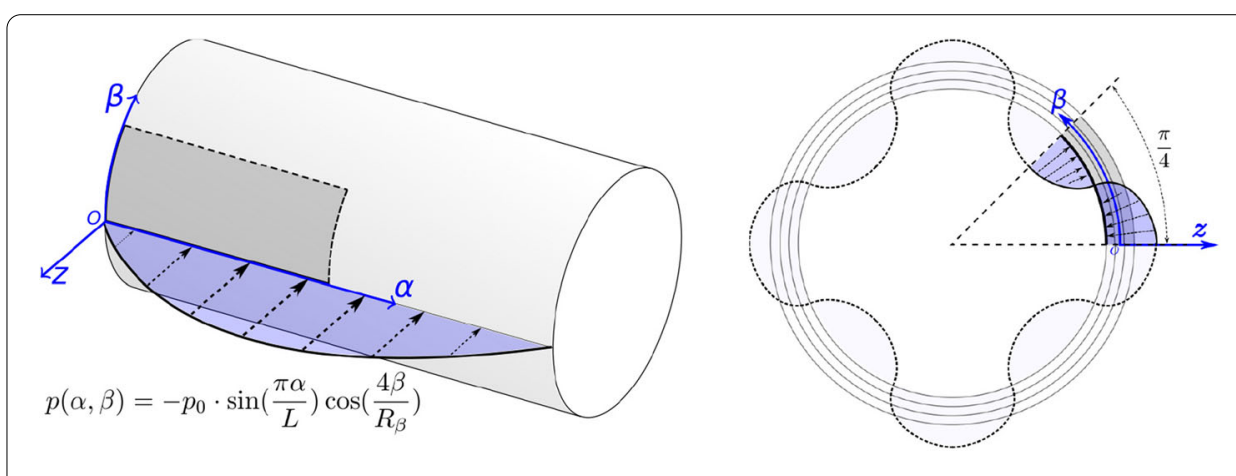

(a) Axial distribution of the pressure

(b) Pressure sectional profile

Fig. 7 The three-layered simply supported cylindrical shells under inner distributed pressure

$$
p(\alpha, \beta)=-p_{0} \sin \frac{\pi \alpha}{L} \cos \frac{4 \beta}{R_{\beta}}
$$

where $L$ is the cylinder length and $R_{\beta}$ the middle surface radius, and $L=4 R_{\beta}$. Figure $7 \mathrm{a}$, $\mathrm{b}$ illustrate the axial variation and the sectional profile of the pressure load, respectively. The material coefficients of the lamina are: $E_{L}=25 E_{T}, G_{L T}=0.5 E_{T}, G_{T T}=0.2 E_{T}$, and $v_{L T}=v_{T T}=0.25$, where the subscripts $L$ and $T$ represent the longitudinal and transverse directions of the fibers, respectively. By taking advantage of the symmetric features in the cylinder axial direction and the cyclic conditions in the circumferential direction, 1/16 of the structure is taken to build the FE model, as indicated by the shaded zone in Fig. 7. The displacement and stress results are non-dimensionalized through:

$$
\begin{aligned}
\bar{w} & =-\frac{10 E_{L} h^{3}}{p_{0} R_{\beta}^{4}} w\left(\frac{L}{2}, 0,0\right), \quad \bar{\sigma}_{\alpha \alpha}=-\frac{10 h^{2}}{p_{0} R_{\beta}^{2}} \sigma_{\alpha \alpha}\left(\frac{L}{2}, 0, \frac{h}{2}\right), \\
\bar{\sigma}_{\beta \beta} & =-\frac{10 h^{2}}{p_{0} R_{\beta}^{2}} \sigma_{\beta \beta}\left(\frac{L}{2}, 0, \frac{h}{2}\right), \quad \bar{\sigma}_{\alpha \beta}=-\frac{10 h^{2}}{p_{0} R_{\beta}^{2}} \sigma_{\alpha \beta}\left(0, \frac{b}{16}, \frac{-h}{2}\right), \\
\bar{\sigma}_{\alpha z} & =-\frac{10 h}{p_{0} R_{\beta}} \sigma_{\alpha z}\left(0,0, \frac{-h}{4}\right), \quad \bar{\sigma}_{\beta z}=-\frac{10 h}{p_{0} R_{\beta}} \sigma_{\beta z}\left(\frac{L}{2}, \frac{b}{16}, \frac{h}{4}\right), \\
\bar{\sigma}_{z z} & =-\frac{1}{p_{0}} \sigma_{z z}\left(\frac{L}{2}, 0, \frac{h}{4}\right) .
\end{aligned}
$$

The 1/16 FE models contain only one element, and the numerical accuracy is improved by increasing the polynomial degree gradually when the relative difference compared to the one-order-lower case is less than $1 \%$ regarding the deflection and stresses as well as the energy components. Considering the load distribution, this benchmark is quite challenging for a single-element model. Table 1 summarizes the converged solutions for each radius-to-thickness ratio value. In general, as the shell structure gets thinner, a higher polynomial degree is required to achieve the desired accuracy. The displacement and stress evaluations obtained with LE4 kinematics agree well with the reference solutions given by Varadan and Bhaskar [48]. The accuracy of the FE results of the thick shell $\left(R_{\beta} / h=2\right)$ can be further improved by refining the thickness functions (TOS), as reported by Li et al. [12]. FSDT leads to good estimation of displacement and in-plane stresses for the thinner shells $\left(R_{\beta} / h=50,100,500\right)$, but fails in other stresses. Also, unlike the LE4 kinematic model, 


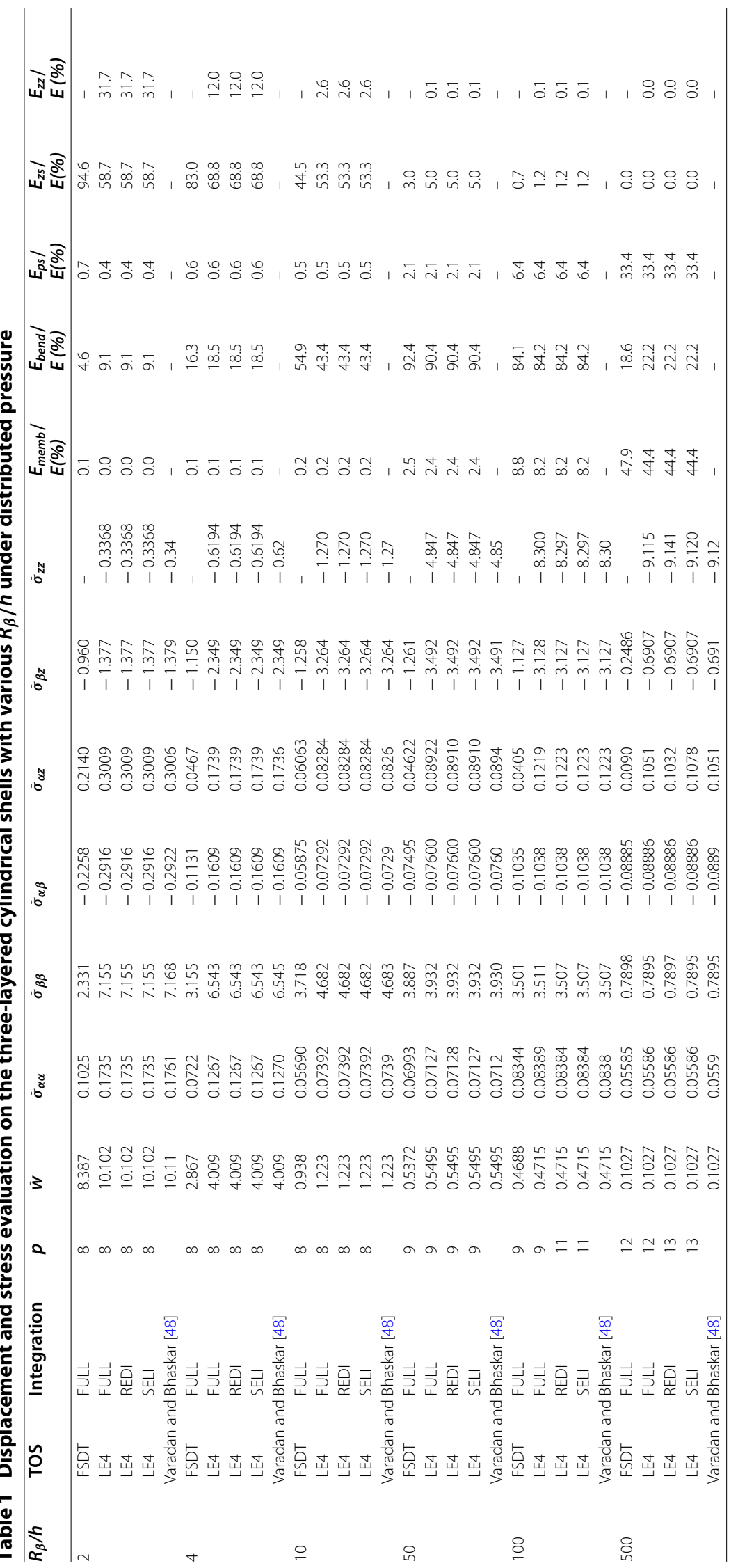




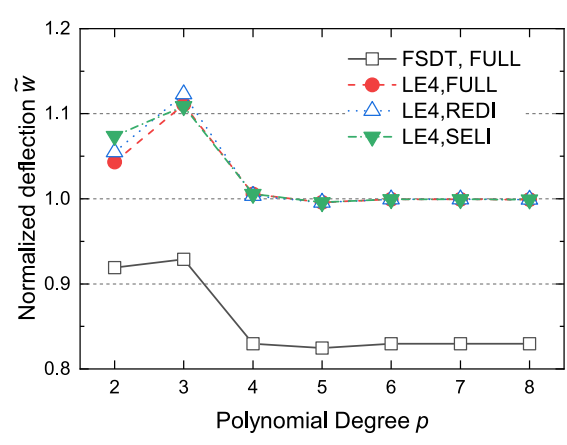

(a) $R_{\beta} / h=2$

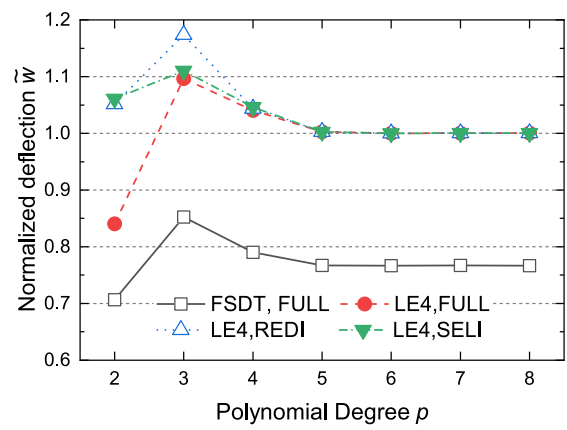

(c) $R_{\beta} / h=10$

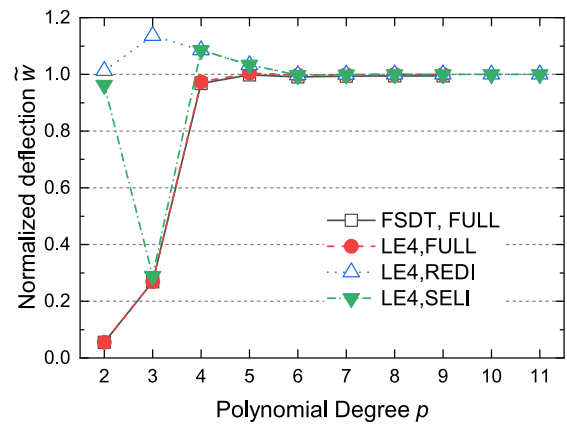

(e) $R_{\beta} / h=100$

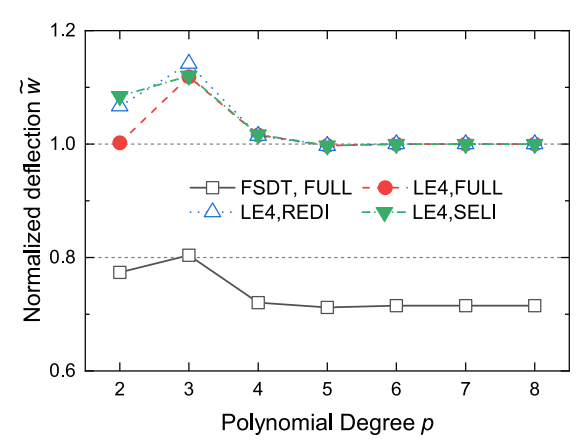

(b) $R_{\beta} / h=4$

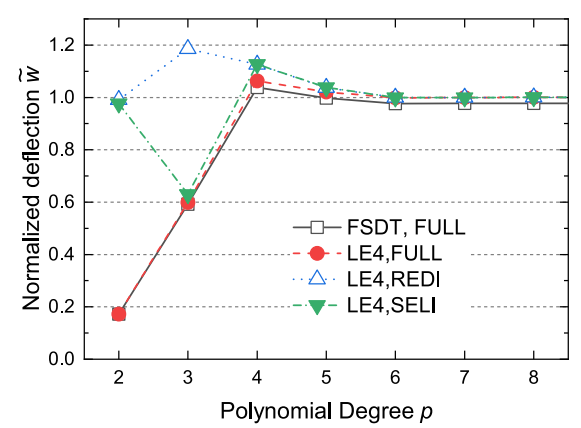

(d) $R_{\beta} / h=50$

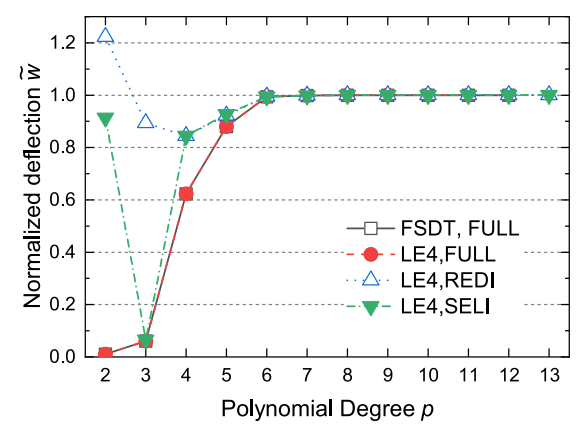

(f) $R_{\beta} / h=500$

Fig. 8 Convergence regarding the normalized deflection of FE models for the three-layered cylindrical shells under distributed pressure, for various radius-to-thickness ratio $R_{\beta} / h$

FSDT ignores the stretch effects in the thickness direction which may play an essential role in thick shells, such as the $R_{\beta} / h=2$ and $R_{\beta} / h=4$ cases in this numerical example.

The convergence of the normalized deflections for each aspect ratio is shown in Fig. 8, in which $\tilde{w}=\bar{w} / \bar{w}_{\text {ref }}$ and $\bar{w}_{\text {ref }}$ is the reference deflection solution. Figure 9 reports the convergence of the FE model regarding the strain energy error, which is calculated by taking the converged solution employing LE4 kinematics with full integration as the reference. It can be observed that for shells with different $R_{\beta} / h$ values, convergence is achieved at different polynomial degrees. Generally, the thinner the shell structure is, the higher polynomial order will be required. Compared to the full integration scheme, the 


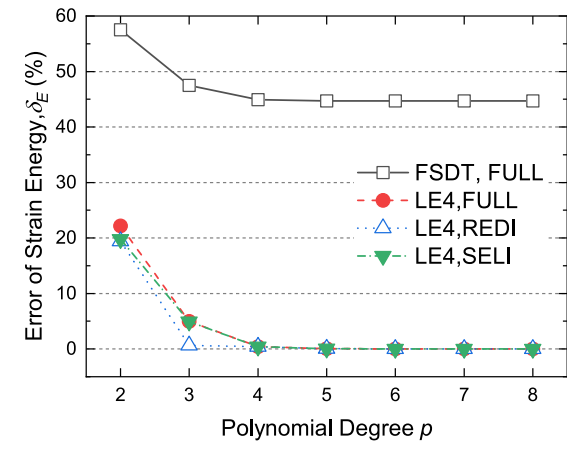

(a) $R_{\beta} / h=2$

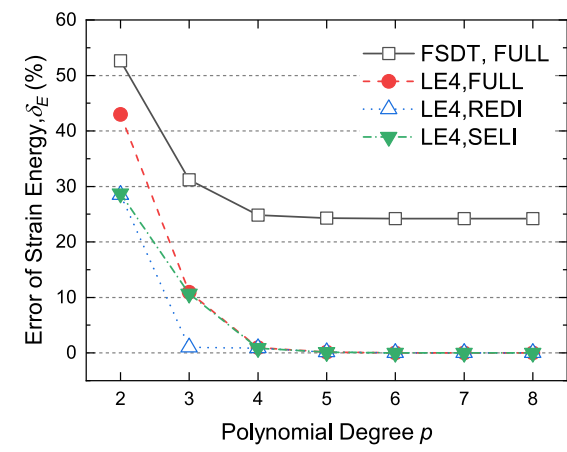

(c) $R_{\beta} / h=10$

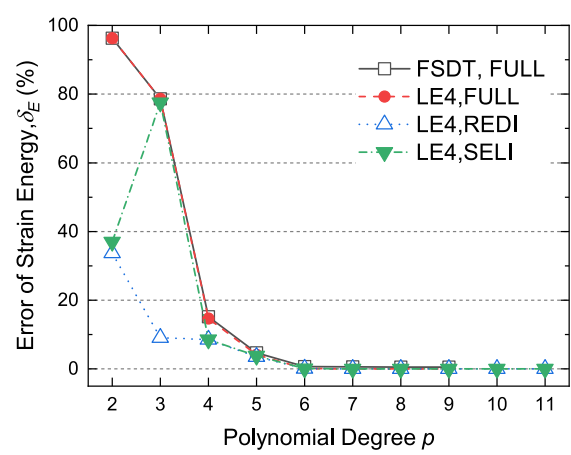

(e) $R_{\beta} / h=100$

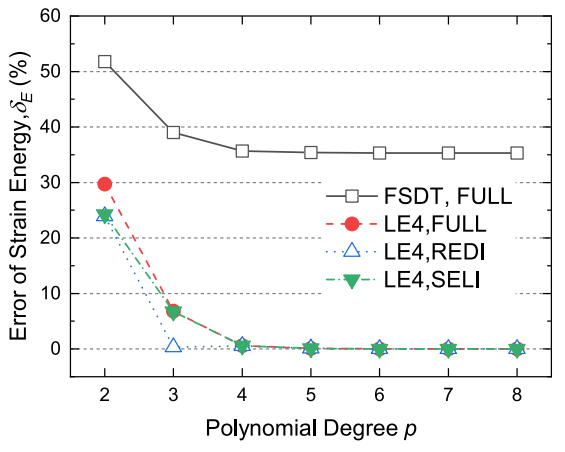

(b) $R_{\beta} / h=4$

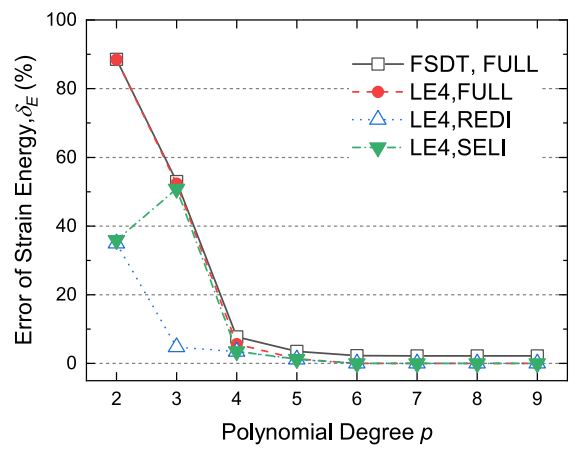

(d) $R_{\beta} / h=50$

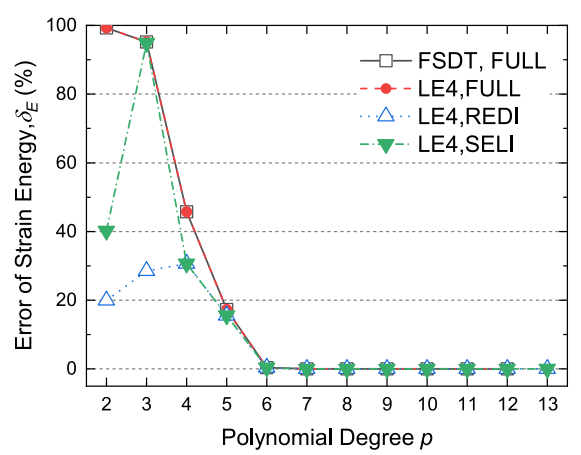

(f) $R_{\beta} / h=500$

Fig. 9 Convergence regarding the strain energy of FE models for the three-layered cylindrical shells under distributed pressure, for various radius-to-thickness ratio $R_{\beta} / \mathrm{h}$

reduced integration technique can help to increase the accuracy in the low-order cases, but the eventual convergence is reached at the same time with full integration. Note that for this single-element model, the detected spurious modes in reduced integrated elements with $p=2$ are not observed to be a significant problem. Notably, the selectively reduced integration improves the accuracy of the single-element FE model with $p=2$ and leads to results quite close to those obtained with full integration in the higher-order cases ( $p \geq 3$ ). When the numerical convergence is reached, all the three kinds of integration schemes lead to results that agree well with the reference solutions. 

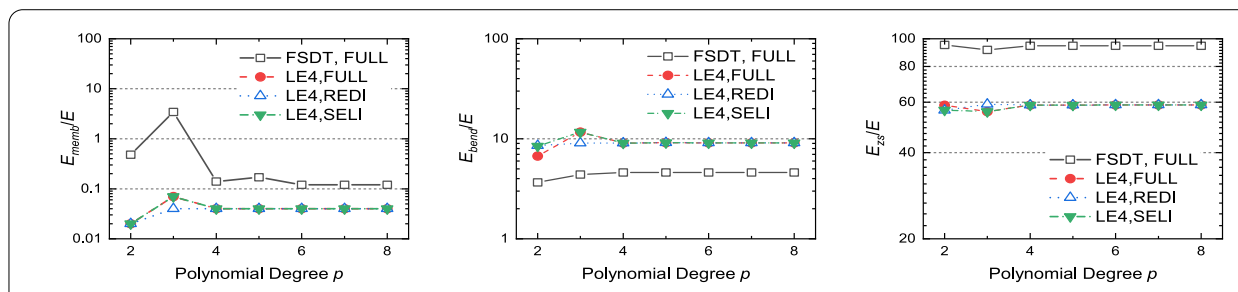

(a) $R_{\beta} / h=2$
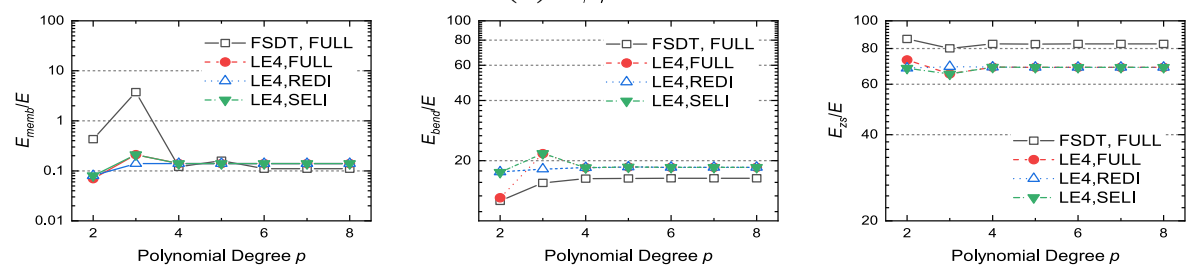

(b) $R_{\beta} / h=4$
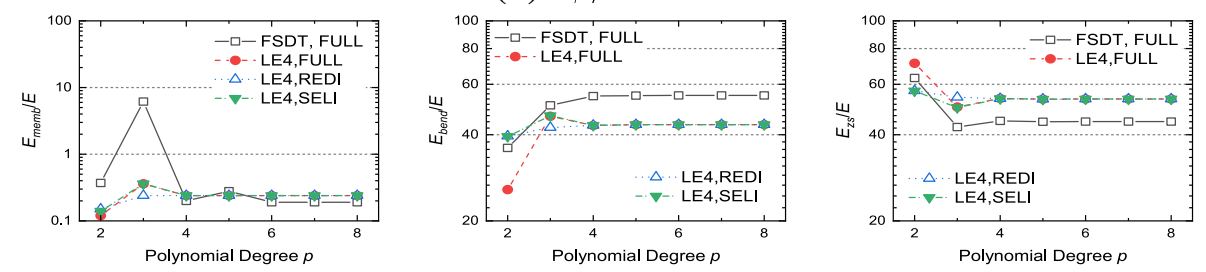

(c) $R_{\beta} / h=10$
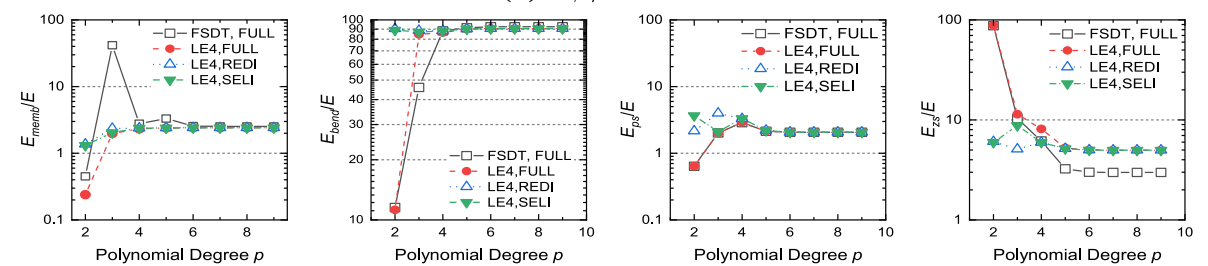

(d) $R_{\beta} / h=50$
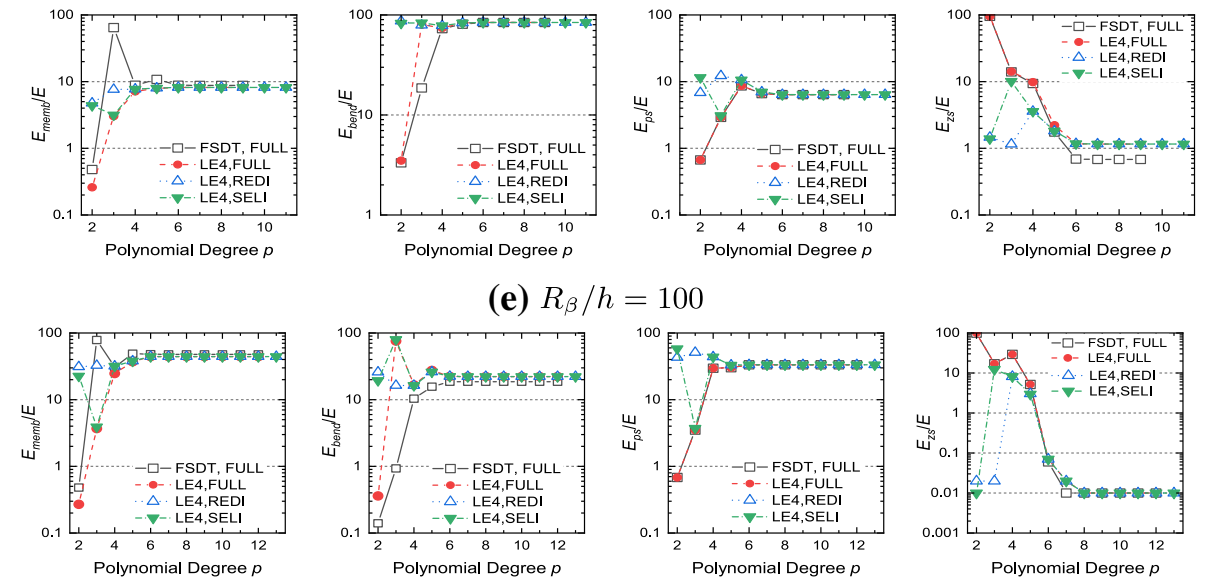

(f) $R_{\beta} / h=500$

Fig. 10 Variation of strain energy components on the three-layered cylindrical shells under distributed pressure with respect to the element polynomial degree, for various radius-to-thickness ratio $R_{\beta} / h$ 


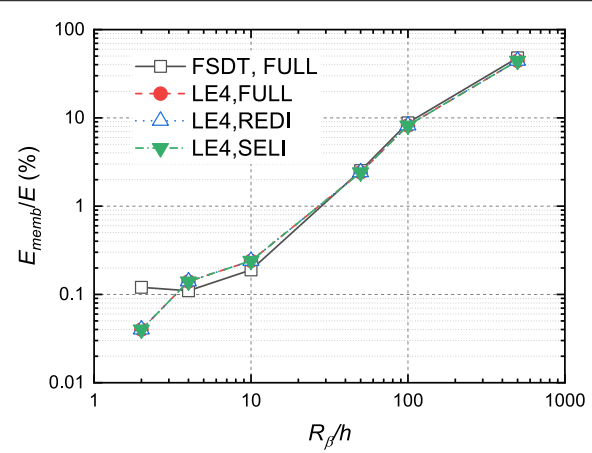

(a) Membrane energy ratio

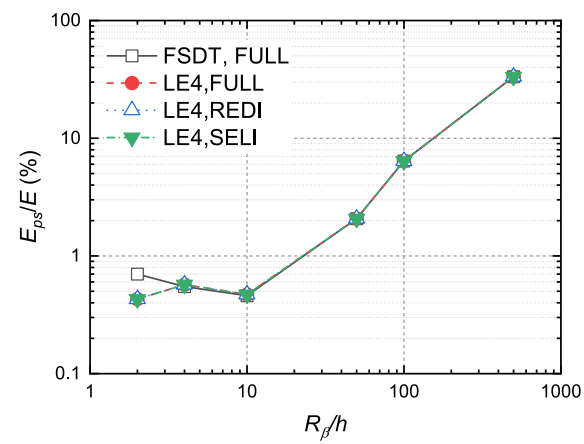

(c) In-plane shear energy ratio

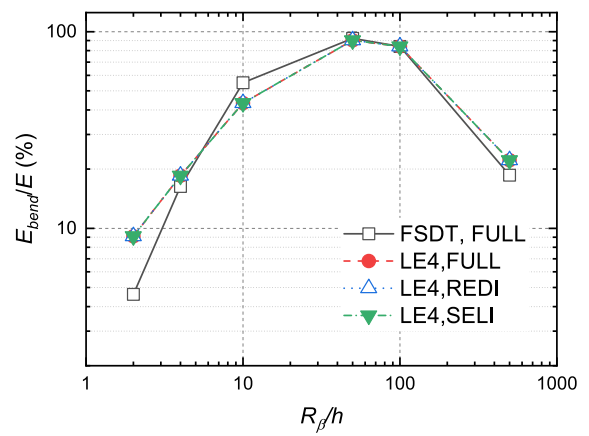

(b) Bending energy ratio

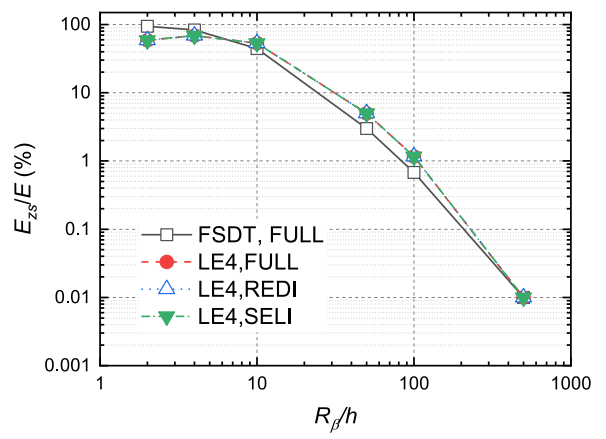

(d) Transverse shear energy ratio

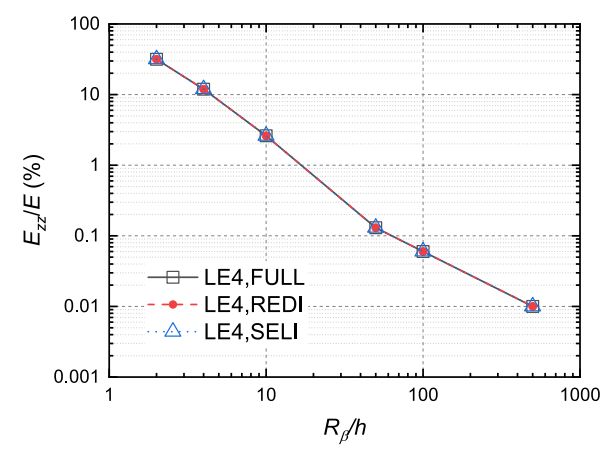

(e) Thickness stretching energy ratio

Fig. 11 Energy components versus radius-to-thickness ratio $R_{\beta} / h$ on the three-layered cylindrical shells under distributed pressure

Figure 10 shows the variation of the ratio of strain energy components with the increase of the polynomial degree of the hierarchical element. Regarding Fig. 10:

- It can be found that for the transverse shear energy, the FSDT and LE4 models with full integration have the same trend.

- For $R_{\beta} / h=2,4$ and 10 , the in-plane shear energy is less than $1 \%$, which can be neglected (see Fig. 11c); as the radius-to-thickness ratio increases, the $E_{p s}$ becomes more significant and is plotted for comparison in Fig. 10.

- The disagreement of FSDT and LE4 in Fig. 10 is due to that the thickness stretch effects are accounted in LE4 model but ignored by FSDT. When the thickness stretch energy is negligible (less than $1 \%$ for $R_{\beta} / h=50,100,500$ ), the transverse shear energy 


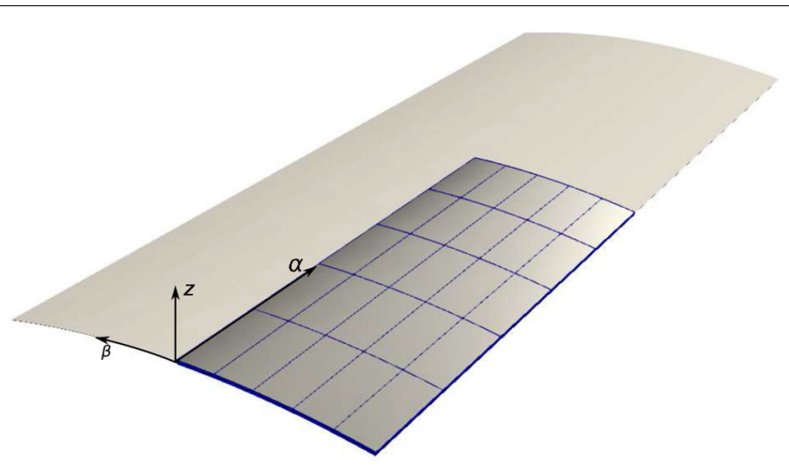

(a) Geometrical features

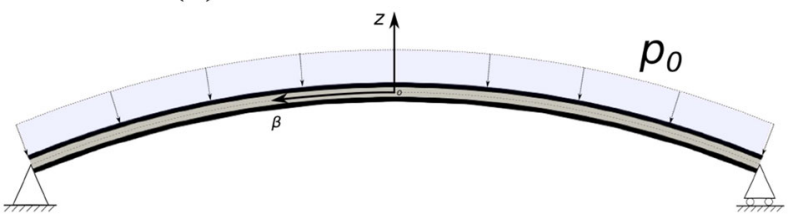

(b) Load and boundary conditions

Fig. 12 Three-layered cylindrical panel under simple supports on two ends

obtained with FSDT and LE4 is quite close, which demonstrates that the kinematic assumptions do not affect the shear locking in thin shells.

- The fully integrated lower-order hierarchical elements $(p=2,3,4)$ suffer from locking on the thinner shells $\left(R_{\beta} / h=50,100,500\right)$, and this locking can be overcome by increasing the polynomial degree $p$ without using any locking-mitigation techniques.

- When the reduced and selective integration schemes are employed, the shear locking phenomenon on the elements with $p=2$ can be greatly alleviated. However, these techniques become less influential when the polynomial degree is further increased, as shown in Fig. 10d-f. Since the newly introduced shape functions lead to improved accuracy, the higher the polynomial degree is, the less necessary the reduced integrated polynomials will become. This effect is more evident for selective integration.

- It should be pointed out that, models with these three integration schemes will converge to comparable solutions when the polynomial order is sufficiently high.

- Figure 10 clearly demonstrates that shear locking is the dominant locking phenomenon for this numerical example.

The variation of the energy components with the radius-to-thickness ratio $R_{\beta} / h$ is summarized in Fig. 11. This variation provides a comprehensive understanding of the structural responses when the shell thickness decreases. It can be observed that the membrane energy ratio keeps increasing monotonically with the reduction of the shell thickness, and the ratios of transverse shear energy and thickness stretch energy decrease and approach zero when the shell is very thin $\left(R_{\beta} / h=100,500\right)$. In general, the energy ratio of the in-plane shear strains increases when the shell gets thinner. The bending energy is significant for moderate-thin shells. To sum up, the transverse strain energy components (transverse shear and thickness stretch) become less dominant with the decrease of the shell thickness. 
Table 2 Displacement and energy evaluation of the three-layered cylindrical panel under bending, obtained through hierarchical elements with $p=7$

\begin{tabular}{lllllllll}
\hline $\boldsymbol{R}_{\boldsymbol{\beta}} / \boldsymbol{h}$ & TOS & Integration & $\overline{\boldsymbol{w}}(\%)-$ & $\boldsymbol{E}_{\boldsymbol{m e m b}} / \boldsymbol{E}(\%)$ & $\boldsymbol{E}_{\text {bend }} / \boldsymbol{E}(\%)$ & $\boldsymbol{E}_{\boldsymbol{p s}} / \boldsymbol{E}(\%)$ & $\boldsymbol{E}_{\mathbf{z s}} / \boldsymbol{E}(\%)$ & $\boldsymbol{E}_{\mathbf{z z}} / \boldsymbol{E}(\%)$ \\
\hline 10 & FSDT & FULL & 294.5 & 0.0 & 20.5 & 0.0 & 79.5 & - \\
& LE4 & FULL & 330.4 & 0.0 & 17.0 & 0.0 & 59.5 & 23.5 \\
& LE4 & REDI & 329.9 & 0.0 & 17.0 & 0.0 & 59.4 & 23.6 \\
& LE4 & SELI & 330.4 & 0.0 & 17.0 & 0.0 & 59.5 & 23.5 \\
100 & FSDT & FULL & 61.67 & 0.0 & 96.3 & 0.0 & 3.7 & - \\
& LE4 & FULL & 62.45 & 0.0 & 95.0 & 0.0 & 5.0 & 0.0 \\
& LE4 & REDI & 62.33 & 0.0 & 95.0 & 0.0 & 5.0 & 0.0 \\
& LE4 & SELI & 62.45 & 0.0 & 95.0 & 0.0 & 5.0 & 0.0 \\
& FSDT & FULL & 59.57 & 0.0 & 100.0 & 0.0 & 0.0 & - \\
& LE4 & FULL & 59.58 & 0.0 & 99.9 & 0.0 & 0.1 & 0.0 \\
& LE4 & REDI & 59.48 & 0.0 & 99.9 & 0.0 & 0.1 & 0.0 \\
& LE4 & SELI & 59.58 & 0.0 & 99.9 & 0.0 & 0.1 & 0.0 \\
& FSDT & FULL & 59.61 & 0.0 & 100.0 & 0.0 & 0.0 & - \\
& LE4 & FULL & 59.61 & 0.0 & 100.0 & 0.0 & 0.0 & 0.0 \\
& LE4 & REDI & 59.52 & 0.0 & 100.0 & 0.0 & 0.0 & 0.0 \\
& LE4 & SELI & 59.61 & 0.0 & 100.0 & 0.0 & 0.0 & 0.0 \\
\hline
\end{tabular}

Simply supported cylindrical panel under bending

This numerical example consists of three-layered cylindrical panels that undergo bending, as shown in Fig. 12. The cylindrical panels have radius $R_{\beta}=10$, mid-surface arch length $b=R_{\beta} \cdot \pi / 20$ in the $\beta$ direction, and length $L=4.0$ along the cylinder axis ( $\alpha$ direction). The radius-to-thickness ratios considered include $R_{\beta} / h=10,100,1000$, and 10,000 . The materials used are the same as in "Three-layered cylindrical shells under distributed pressure" section. The lamination sequence is $\left(0^{\circ} / 90^{\circ} / 0^{\circ}\right)$, and the thicknesses of the three layers are $\frac{h}{4}, \frac{h}{2}$, and $\frac{h}{4}$, separately. As illustrated in Fig. 12b, the cylindrical panels are simply supported on the two ends along the cylinder axis, and free on the other two edges. The simple supports follow:

$$
\beta=-\frac{b}{2}, \frac{b}{2}: \quad u=0, w=0
$$

The structure is imposed to constant pressure load $p_{0}$ on the top surface. The vertical displacement $w$ is non-dimensionalized through the following parameters:

$$
\bar{w}=-\frac{10^{4} E_{L} h^{3}}{p_{0} R_{\beta}^{4}} w\left(\frac{L}{2}, 0,0\right)
$$

By making use of the symmetric features of the boundary conditions, a 1/4 FE model with one rectangular hierarchical element is employed, as demonstrated in Fig. 12a. The one-element model is refined by increasing its polynomial degree of the hierarchical shape functions until the relative difference of two neighboring orders is less than $0.5 \%$ regarding the displacement evaluation $\bar{w}$. On the whole, $p=7$ is sufficient to guarantee the convergence. The displacement evaluation $\bar{w}$ and strain components estimation obtained through hierarchical elements with $p=7$ are listed in Table 2 .

Figures 13 and 14 summarize the convergence of the FE models with the increase of the polynomial degree concerning the normalized displacement evaluation and error of 


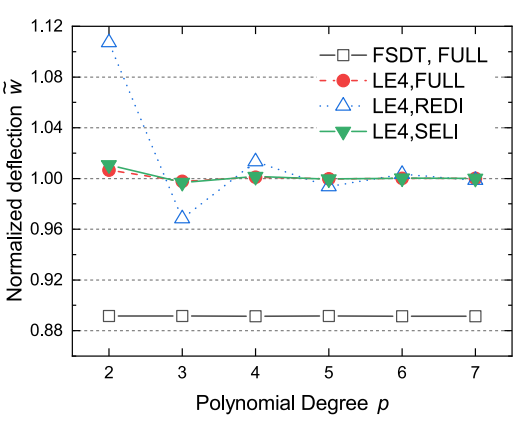

(a) $R_{\beta} / h=10$

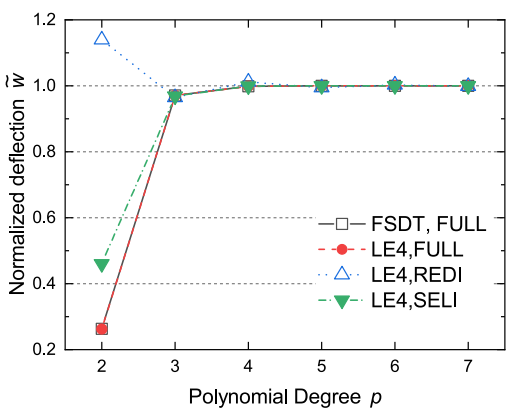

(c) $R_{\beta} / h=1000$

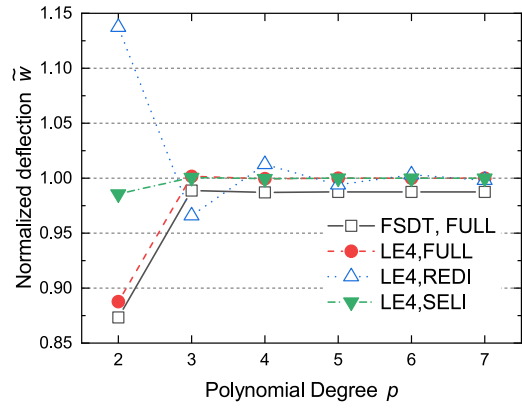

(b) $R_{\beta} / h=100$

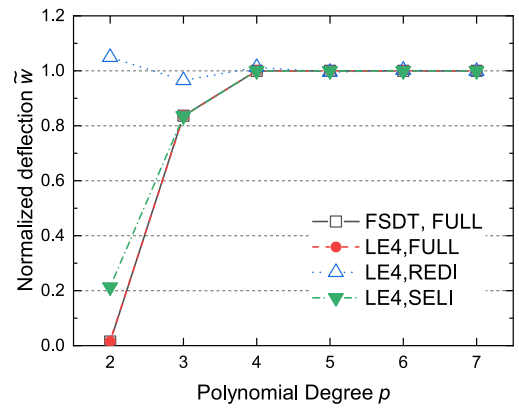

(d) $R_{\beta} / h=10000$

Fig. 13 Convergence regarding the normalized deflection of FE models for the simply supported cylindrical panels under bending, for various radius-to-thickness ratio $R_{\beta} / h$

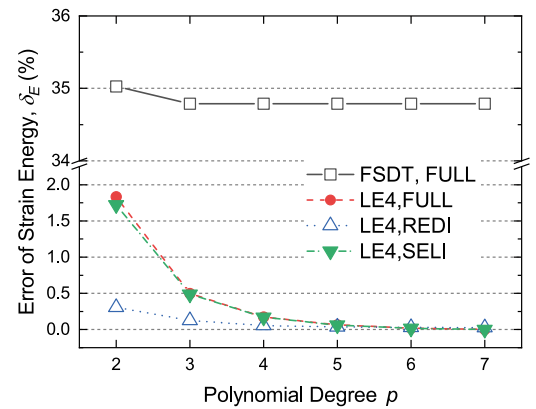

(a) $R_{\beta} / h=10$

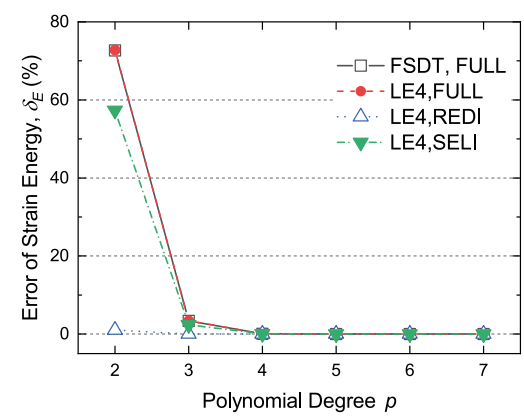

(c) $R_{\beta} / h=1000$

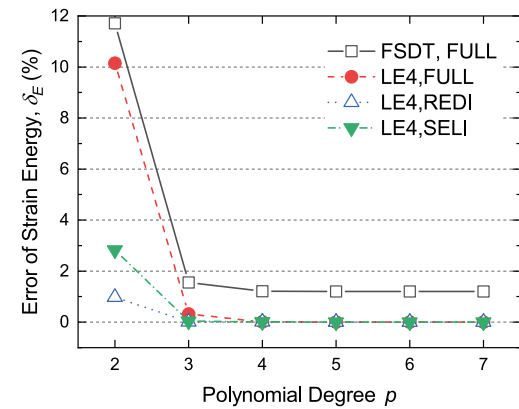

(b) $R_{\beta} / h=100$

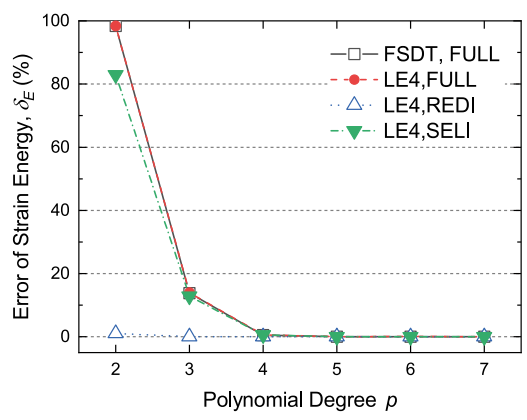

(d) $R_{\beta} / h=10000$

Fig. 14 Convergence regarding the strain energy of FE models for the simply supported cylindrical panels under bending, for various radius-to-thickness ratio $R_{\beta} / h$ 


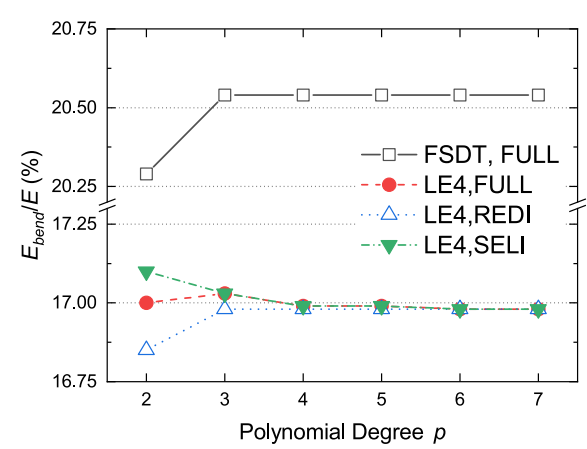

(a) $R_{\beta} / h=10$

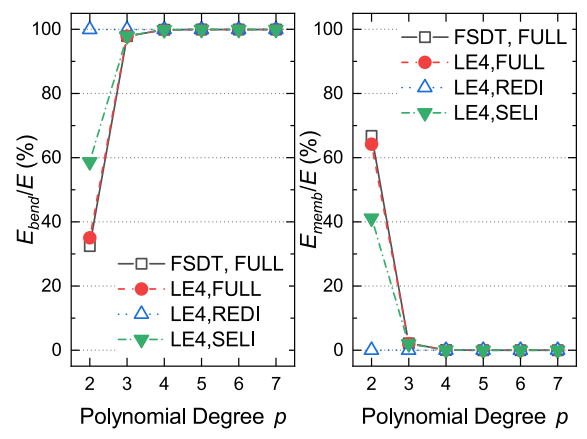

(c) $R_{\beta} / h=1000$
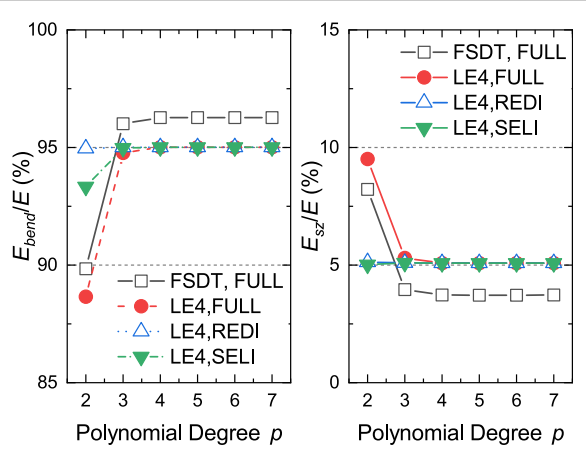

(b) $R_{\beta} / h=100$
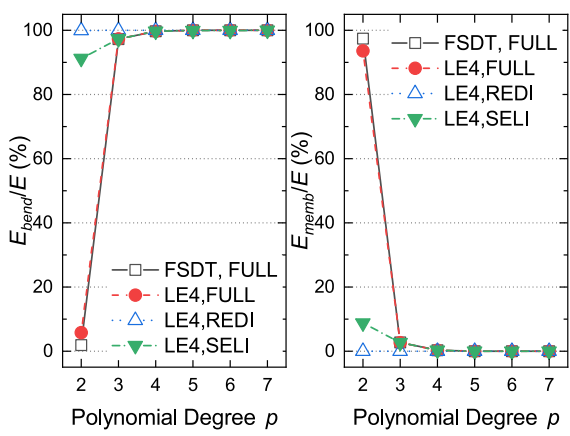

(d) $R_{\beta} / h=10000$

Fig. 15 Variation of relevant energy components for each aspect ratio with respect to the polynomial degree of FE models for the three-layered cylindrical panel under bending, for various radius-to-thickness ratio $R_{\beta} / h$

strain energy, respectively. Since no reference is available in the literature, the reference solutions are given by FE models with full integration and $p=8$. It can be observed that for the relative thick shells with $R_{\beta} / h=10$ and 100 , the reduced integration leads to less accurate displacement estimation and lower convergence compared with full and selective integration schemes. Meanwhile, for the thinner shells with $R_{\beta} / h=1000$ and 10,000, reduced integration models give better accuracy than the other two integration schemes in the lower-order hierarchical shell elements $\left(p=2\right.$ for $R_{\beta} / h=1000$, and $p=2,3$ for $\left.R_{\beta} / h=10,000\right)$. It seems that reduced integration "unnecessarily" leads to over-soft bending stiffness when no noticeable locking is present in this numerical example. The displacement and energy estimations obtained through selective integration show almost no difference from those of full integration when the element order $p \geq 4$.

The variation of the relevant energy terms with respect to the element polynomial degree is reported in Fig. 15. For the thick shells with $R_{\beta}=10$, no apparent locking is observed on the low-order hierarchical elements. As the shell becomes thinner, locking becomes significant on low-order elements. For the moderate-thick shell with $R_{\beta} / h=100$, locking occurs on the fully integrated elements for $p=2$ and it decreases rapidly as the polynomial degree goes higher. This locking is mainly shear locking, which can be observed through the comparison of bending energy and transverse shear energy in Fig. 15b. On the other hand, for the thin shells with $R_{\beta} / h=1000$ and 10,000, significant membrane locking emerges on the low-order element $(p=2,3)$ with full integration, which also drops rapidly with the increase of the polynomial degree. For all the relatively thin shells $\left(R_{\beta} / h=\right.$ 


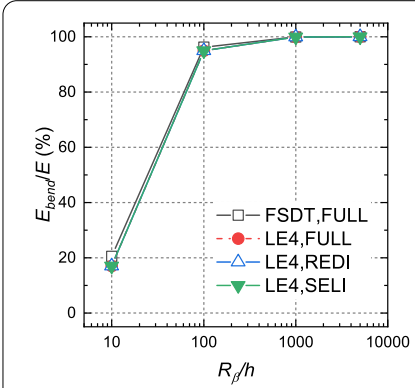

(a) $E_{\text {bend }} / E$

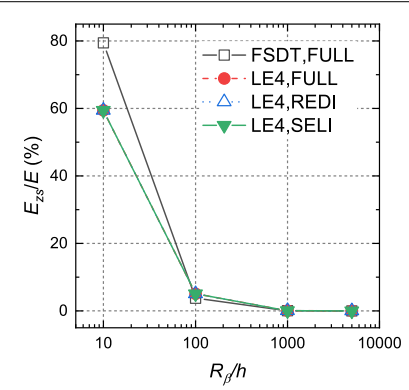

(b) $E_{z s} / E$

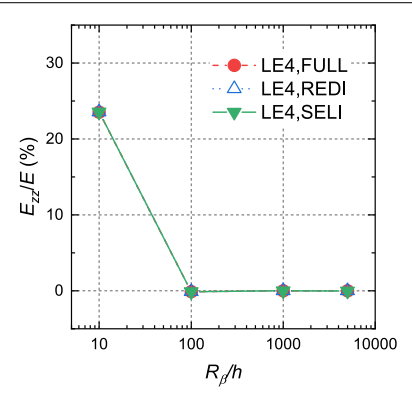

(c) $E_{z z} / E$

Fig. 16 Ratio of different energy components versus radius-to-thickness ratio on the three-layered cylindrical panel under bending

100,1000 , and 10,000 ), reduced integration is able to mitigate the membrane locking effectively which occurs on low-order hierarchical elements, as shown in Fig. 15b-d. The selective integration technique also leads to improved energy estimation, yet less effective compared to reduced integration in this case. To sum up, when the numerical convergence is achieved via $p$-refinement, all the three integration schemes give equivalent locking-free solutions.

From Table 2, it can also be observed that when the hierarchical elements are sufficiently refined, all the integration schemes give results in considerable agreement with each other. The variation of different energy components concerning the increase of the element polynomial order is plotted in Fig. 16. As expected, the strain energy components due to the out-of-plane strains, namely the transverse shear strain energy and thickness stretch energy, decrease rapidly with the increase of the radius-to-thickness ratio and approach zero on thin shells. Also, it can be found that the membrane energy and in-plane shear energy are absent in this numerical example. On the thin shells with $R_{\beta} / h=1000$ and 10,000 , the strain energy contains only the bending component.

\section{Conclusions}

In this article, the performance of hierarchical elements in the numerical analysis of laminated shell structures is assessed. The numerically efficient integration schemes for hierarchical shell elements are discussed. Proper energy decomposition is proposed and adopted in the evaluation of shear and membrane locking phenomena. Through the numerical investigation with single-element $p$-version finite element models, the following conclusions can be drawn:

- Hierarchical shell elements with full integration are capable of overcoming both shear and membrane locking via plain $p$-refinement for structures with an aspect ratio in a wide range without using special locking mitigation techniques;

- Hierarchical shell elements with polynomial degree $p \geq 3$ employing reduced integration scheme and $p \geq 2$ adopting selective integration technique are robust;

- Reduced and selectively reduced integration schemes are helpful with the lower-order hierarchical elements to alleviate the shear and membrane locking phenomena; 
- When numerical convergence is reached with a higher-order polynomial degree, the three integration schemes, namely the full, reduced and selective integration, lead to the same accuracy;

- With hierarchical shell elements, the physically zero transverse shear strain energy on thin shells can be achieved numerically.

The above evaluation demonstrates the high efficiency of hierarchical shell elements in the analysis of laminated shell structures. Only rectangular elements are tested in the present article. Distorted meshes should also be considered in the future.

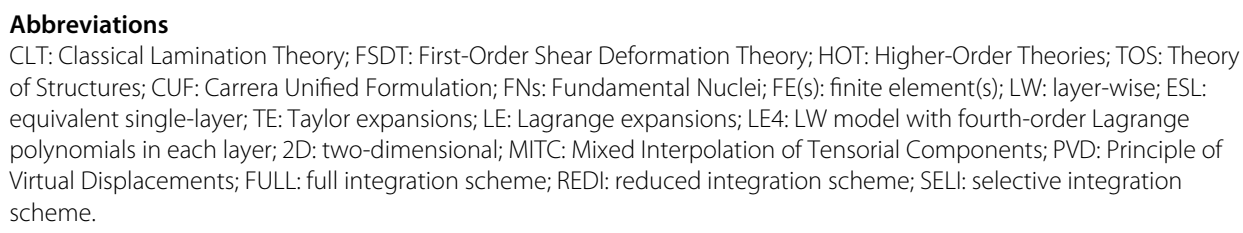
of Structures; CUF: Carrera Unified Formulation; FNs: Fundamental Nuclei; FE(s): finite element(s); LW: layer-wise; ESL: equivalent single-layer; TE: Taylor expansions; LE: Lagrange expansions; LE4: LW model with fourth-order Lagrange polynomials in each layer; 2D: two-dimensional; MITC: Mixed Interpolation of Tensorial Components; PVD: Principle of Virtual Displacements; FULL: full integration scheme; REDI: reduced integration scheme; SELI: selective integration scheme.

\section{Authors' contributions}

The adopted methodology, Carrera Unified Formulation, was initially envisaged and formulated by EC. EC and MC formulated the refined multi-layered shell models in CUF framework. EZ and GL developed the finite element tools. GL conceived the idea, collected and interpreted the numerical results, and was the major contributor in writing the manuscript. AGM, MC, AP, and GMK provided valuable suggestions. All authors read and approved the final manuscript.

\section{Author details}

${ }^{1}$ MUL2 Group, Department of Mechanical and Aerospace Engineering, Politecnico di Torino, Corso Duca degli Abruzzi 24, 10129 Turin, Italy, ${ }^{2}$ Laboratory of Intelligent Materials and Structures, Tambov State Technical University, Sovetskaya Street 106, 392000 Tambov, Russia.

\section{Competing interests}

The authors declare that they have no competing interests.

\section{Funding}

This research work has been carried out within the project FULLCOMP (FULLy analysis, design, manufacturing, and health monitoring of COMPosite structures), funded by the European Union Horizon 2020 Research and Innovation program under the Marie Skłodowska Curie Grant Agreement No. 642121. EC and GMK acknowledge the Russian Science Foundation under Grant No. 18-19-00092.

\section{Publisher's Note}

Springer Nature remains neutral with regard to jurisdictional claims in published maps and institutional affiliations.

Received: 1 November 2018 Accepted: 16 April 2019

Published online: 24 May 2019

\section{References}

1. KoiterWT. On the foundations of the linear theory of thin elastic shell. Proc Kon Nederl Akad Wetensch. 1970;73(3):169_ 95.

2. Ciarlet PG, Gratie L. Another approach to linear shell theory and a new proof of Korn's inequality on a surface. Comptes Rendus Mathematique. 2005;340(6):471-8.

3. Reissner E, Stavsky Y. Bending and stretching of certain types of heterogeneous aeolotropic elastic plates. J Appl Mech. 1961:28(3):402-8

4. Reissner $E$. The effect of transverse shear deformation on the bending of elastic plates. J Appl Mech. 1945;12:A69-77.

5. Mindlin RD. Influence of rotatory inertia and shear on flexural motions of isotropic, elastic plates. J Appl Mech. $1951 ; 18: 31-8$

6. Reddy JN. A simple higher-order theory for laminated composite plates. J Appl Mech. 1984;51(4):745-52.

7. Reddy JN. Mechanics of laminated composite plates and shells: theory and analysis. New York: CRC Press; 2004.

8. Palazotto AN. Nonlinear analysis of shell structures. AIAA Series. 1992.

9. Carrera E. Theories and finite elements for multilayered, anisotropic, composite plates and shells. Arch Comput Methods Eng. 2002;9(2):87-140.

10. Cinefra M, Carrera E. Shell finite elements with different through-the-thickness kinematics for the linear analysis of cylindrical multilayered structures. Int J Numer Methods Eng. 2013;93(2):160-82.

11. Cinefra M, Valvano S. A variable kinematic doubly-curved MITC9 shell element for the analysis of laminated composites. Mech Adv Mater Struct. 2016;23(11):1312-25.

12. Li G, Carrera E, Cinefra M, de Miguel AG, Pagani A, Zappino E. An adaptable refinement approach for shell finite element models based on node-dependent kinematics. Compos Struct. 2019;210:1-19. 
13. Zienkiewicz OC, Taylor RL, Too JM. Reduced integration technique in general analysis of plates and shells. Int J Numer Methods Eng. 1971;3(2):275-90.

14. Stolarski H, Belytschko T. Shear and membrane locking in curved CO elements. Comput Methods Appl Mech Eng. 1983;41(3):279-96.

15. Belytschko T, Stolarski H, Liu WK, Carpenter N, Ong Jame SJ. Stress projection for membrane and shear locking in shell finite elements. Comput Methods Appl Mech Eng. 1985;51(1-3):221-58.

16. Hughes TJR, Cohen M, Haroun M. Reduced and selective integration techniques in the finite element analysis of plates. Nuclear Eng Design. 1978:46(1):203-22.

17. Zienkiewicz OC, Nakazawa S. On variational formulation and its modifications for numerical solution. Comput Struct. 1984;19(1-2):303-13.

18. Zienkiewicz OC, Taylor RL. The finite element method for solid and structural mechanics. New York: Elsevier; 2005.

19. Dvorkin EN, Bathe K-J. A continuum mechanics based four-node shell element for general non-linear analysis. Eng Comput. 1984;1 (1):77-88.

20. Bathe K-J, Dvorkin EN. A four-node plate bending element based on Mindlin/Reissner plate theory and a mixed interpolation. Int J Numer Methods Eng. 1985;21(2):367-83.

21. Bucalem ML, Bathe K-J. Higher-order MITC general shell elements. Int J Numer Methods Eng. 1993;36(21):3729-54.

22. Bathe K-J, losilevich A, Chapelle D. An evaluation of the MITC shell elements. Comput Struct. 2000;75(1):1-30.

23. Carrera Erasmo, Parisch Horst. An evaluation of geometrical nonlinear effects of thin and moderately thick multilayered composite shells. Compos Struct. 1997;40(1):11-24.

24. Babuška I, Narasimhan R. The Babuška-Brezzi condition and the patch test: an example. Comput Methods Appl Mech Eng. 1997;140(1-2):183-99.

25. Carrera E, Cinefra M, Nali P. MITC technique extended to variable kinematic multilayered plate elements. Compos Struct. 2010;92(8):1888-95.

26. Carrera E, Cinefra M, Li G, Kulikov GM. MITC9 shell finite elements with miscellaneous through-the-thickness functions for the analysis of laminated structures. Compos Struct. 2016;154:360-73.

27. Carrera E, Cinefra M, Li G. Refined finite element solutions for anisotropic laminated plates. Compos Struct. 2018;183:63-76.

28. Carrera E, de Miguel AG, Pagani A. Extension of MITC to higher-order beam models and shear locking analysis for compact, thin-walled, and composite structures. Int J Numer Methods Eng. 2017;112(13):1889-908.

29. Ko Y, Lee P-S, Bathe K-J. The MITC4+ shell element and its performance. Comput Struct. 2016;169:57-68.

30. Ko Yeongbin, Lee Phill-Seung, Bathe Klaus-Jürgen. A new 4-node mitc element for analysis of two-dimensional solids and its formulation in a shell element. Comput Struct. 2017;192:34-49.

31. Bathe K-J. Finite element procedures. Klaus-Jurgen Bathe. 2006.

32. Suri M. Analytical and computational assessment of locking in the hp finite element method. Comput Methods Appl Mech Eng. 1996;133(3-4):347-71.

33. Schwab C. P-and Hp-finite element methods: theory and applications in solid and fluid mechanics (numerical mathematics and scientific computation). New York: Oxford University Press; 1999.

34. Düster $A$, Bröker $H$, Rank E. The p-version of the finite element method for three-dimensional curved thin walled structures. Int J Numer Methods Eng. 2001;52(7):673-703.

35. Szabó B, Düster A, Rank E. The p-version of the finite element method. New York: Wiley; 2004.

36. Scapolla T, Croce LD. Combining hierarchic high order and mixed-interpolated finite elements for Reissner-Mindlin plate problems. Comput Methods Appl Mech Eng. 1994;116(1-4):185-92.

37. Scapolla T, Croce LD. Hierarchic and mixed-interpolated finite elements for Reissner-Mindlin problems. Commun Numer Methods Eng. 1995;11(7):549-62.

38. Chinosi C, Della Croce L, Scapolla T. Hierarchic finite elements for thin Naghdi shell model. Int J Solids Struct. 1998;35(16):1863-80.

39. Della Croce Lucia, Scapolla Terenzio. Hierarchic finite elements with selective and uniform reduced integration for Reissner-Mindlin plates. Comput Mech. 1992;10(2):121-31.

40. Szabó Barna A. Mesh design for the p-version of the finite element method. Comput Methods Appl Mech Eng. 1986;55(1-2):181-97.

41. Szabó BA, Babuška I. Finite element analysis. New York: Wiley; 1991.

42. Li G, Miguel AG, Pagani A, Zappino E, Carrera E. Finite beam elements based on Legendre polynomial expansions and node-dependent kinematics for the global-local analysis of composite structures. Eur J Mech A Solids. 2018b;74:11223.

43. Zappino E, Li G, Pagani A, Carrera E, de Miguel Alberto G. Use of higher-order Legendre polynomials for multilayered plate elements with node-dependent kinematics. Compos Struct. 2018;202:222-32 Special issue dedicated to lan Marshall.

44. Leissa AW. Vibration of shells, vol 288. Scientific and Technical Information Office, National Aeronautics and Space Administration Washington; 1973.

45. Jones RM. Buckling of bars, plates, and shells. Blacksburg: Bull Ridge Corporation; 2006.

46. Carrera E, Cinefra M, Petrolo M, Zappino E. Finite element analysis of structures through Unified Formulation. New York: Wiley; 2014.

47. Naghdi PM. The theory of plates and shells. Handbuch der Physik. 1972;6:425-640.

48. Varadan TK, Bhaskar K. Bending of laminated orthotropic cylindrical shells-an elasticity approach. Compos Struct. 1991;17(2):141-56. 